\title{
Tectorigenin alleviates intrahepatic cholestasis by inhibiting hepatic inflammation and bile accumulation via activation of PPARg
}

\author{
Jiaqing Xiang ${ }^{1}$, Guangyan Yang ${ }^{1}$, Chuanrui $\mathrm{Ma}^{2}$, Linlin $\mathrm{Wei}^{3}$, $\mathrm{Han}_{\mathrm{Wu}}{ }^{1}$, Xiuhua $\mathrm{Tao}^{3}$, \\ Lingyun Jiang ${ }^{1}$, Zhen Liang ${ }^{1}$, Lin Kang ${ }^{1}$, and shu yang ${ }^{1}$ \\ ${ }^{1}$ Shenzhen People's Hospital \\ ${ }^{2}$ First Teaching Hospital of Tianjin University of Traditional Chinese Medicine \\ ${ }^{3}$ Jiangxi Academy of Agricultural Sciences
}

September 11, 2020

\begin{abstract}
Background and purpose: Increasing evidence has shown that human cholestasis is closely related to hepatic macrophage accumulation and activation. Research has indicated that peroxisome proliferator-activated receptor-g (PPARg) activation exerts liver protection in cholestatic liver disease (CLD), particularly by ameliorating inflammation and fibrosis, thus limiting disease progression. However, existing PPARg agonists, such as troglitazone and rosiglitazone, have significant side effects that impede their clinical application in the treatment of CLD. In this study, we found that tectorigenin (TEC) can alleviate intrahepatic cholestasis in mice by activating PPARg. Experimental approach: Wild-type mice received intragastric administration of a-naphthylisothiocyanate (ANIT) or were fed a diet containing 0.1\% 3,5-diethoxycarbonyl-1,4-dihydrocollidine (DDC) to establish an experimental intrahepatic cholestasis model and TEC intervention simultaneously, followed by determination of intrahepatic cholestasis and the involved mechanisms. In addition, PPARg deficient mice were administered ANIT and/or TEC to determine whether TEC exerts its liver protection effect by activating PPARg. Key results: Our results demonstrated that TEC intervention alleviated intrahepatic cholestasis by inhibiting hepatic macrophage recruitment and activation as well as promoting the expression of bile transporters through activating PPARg. Furthermore, our results show that TEC increased bile salt export pump (Bsep) expression through enhanced PPARg binding to the Bsep promoter. We also demonstrated that PPARg deficiency blocked the hepatocyte protective effect of TEC during cholestasis. Conclusions and implications: In conclusion, TEC reduced hepatic macrophage recruitment and activation, and enhanced bile acid export by activating PPARg. Taken together, our results suggest that TEC is a potential drug for the prevention of CLD.
\end{abstract}

\section{Lay summary}

Intrahepatic cholestasis includes primary biliary cholangitis and primary sclerosing cholangitis. It can cause fibrosis, cirrhosis and eventually liver failure. Effective drugs for PBC and PSC are still limited and new treatment strategies are urgently needed. This study defines tectorigenin, an isoflavone, have the potential to alleviate cholestatic liver disease, which is achieved mainly by relieving liver inflammation and promoting the secretion of bile in hepatocyte.

\section{Graphical Abstract}




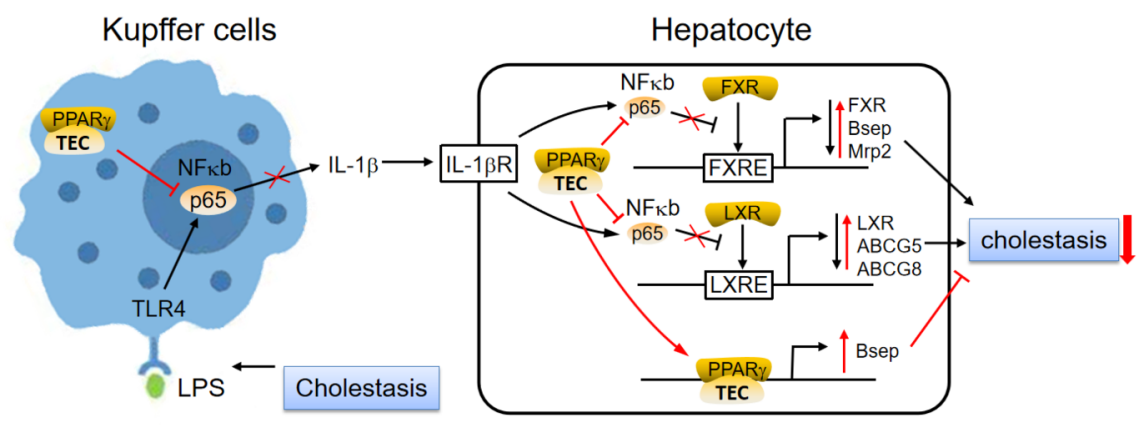

Black line indicated the pathogenesis of cholestasis

Red line represented the mechanism by which TEC alleviates cholestasis

TEC alleviate CLD by activating PPAR $\gamma$, and its molecular mechanisms are mainly as follows: 1) restrain macrophage activation and its derived inflammatory factor, such as IL-1 $\beta$ - 2) upregulated the expression of FXR and LXR as well as FXR-regulated and LXR-regulated genes through activating PPAR $\gamma$ to block NF «b-p65 mediated inhibition of FXR and LXR in hepatocyte; 3) enhanced the PPAR $\gamma$ binding to Bsep promoter and increased its expression.

\section{Introduction}

Cholestatic liver disease (CLD) manifests as a multitude of etiological heterogeneous hepatobiliary disorders, mainly including primary biliary cholangitis (PBC) and primary sclerosing cholangitis (PSC), in adults. CLD, which is also called cholestasis, describes a range of conditions caused by the accumulation of bile acids in the liver, resulting in hepatocellular necrosis, apoptosis, progressive fibrosis, and even end-stage liver disease ${ }^{[1]}$.

Accumulated evidence has proved that ursodeoxycholic acid (UDCA) can decrease the progression of $\mathrm{PBC}^{[2]}$; however, approximately one-third of patients do not respond to UDCA treatment ${ }^{[3]}$. In 2016, obeticholic acid (OCA), a farnesoid X receptor (FXR) agonist, was found could decrease serum alkaline phosphatase (AP) level in CLD patient, was approved by the FDA for UDCA nonresponders ${ }^{[4,5]}$. In spite of this, long-term follow-up is needed to confirm the safety and effectiveness of this novel treatment. At present, there is no clinical evidence that there is any medical therapy can alter the course of PSC. Current treatment regimens focus on symptom management and treatment of cholangitis ${ }^{[6]}$. Effective drugs for PBC and PSC are still limited and new treatment strategies are urgently needed.

Increasing evidence shows that human cholestasis is closely related to the disorder of microbiome composition $^{[7,8]}$, increased intestinal permeability, enhanced translocation of pathogenic bacteria and bacterial toxins, such as lipopolysaccharide (LPS) into the liver ${ }^{[9,10]}$. Inflammasomes and proinflammatory cytokines are then activated due to these microbe-derived products, which are recognized by the innate immune system via pathogen recognition receptors (TLRs and NLRs) ${ }^{[11]}$. When persistent liver inflammation is unresolved, the proinflammatory milieu can play a detrimental role in parenchymal and nonparenchymal liver cells resulting in fibrosis and ultimately loss of function ${ }^{[12]}$.

Macrophages, which are composed of resident tissue macrophages and monocyte-derived recruited cells, can differentiate into either classically activated macrophages (a pro-inflammatory phenotype, also called M1 polarity) or alternatively activated macrophages (M2 polarity) which express anti-inflammatory cytokines ${ }^{[13]}$. Recent studies have demonstrated that the intestinal microbiome leads to the process of cholestasis-mediated cell death and inflammation by activating the mechanisms of the inflammasome in macrophages ${ }^{[14]}$. Additionally, clinical studies have suggested that in cholestasis patients, the recruitment of monocytes and macrophages in diseased liver is significantly increased ${ }^{[15]}$. The expression of various monocyte chemotactic proteins, such as monocyte chemoattractant protein (MCP)-1 is significantly increased in the livers of pa- 
tients with cholestasis ${ }^{[16]}$. In parallel to these findings, $\mathrm{C}-\mathrm{C}$ chemokine receptor type-2 (CCR-2) expressed by liver macrophages is accompanied by increasing macrophage numbers in the livers of cholestasis patients ${ }^{[17]}$.

Importantly, macrophages in CLD patients are more susceptible to stimulation signals such as endotoxin (for example, LPS), which have also been shown to be increased in cholestasis patients ${ }^{[18]}$. In line with the above findings, the expression and activity of TLR4 (the primary receptor of LPS) are upregulated on the monocytes of CLD patients, leading to LPS hyperreactivity and increased production of proinflammatory cytokines [i.e., interleukin (IL)-1 $\beta$, IL-6, and IL-8] ${ }^{[19]}$. It is reported that macrophage-derived IL- $1 \beta$ is a key cytokine activating hepatocyte nuclear factor $\chi \mathrm{B}(\mathrm{NF} \varkappa \mathrm{b})$, which is the master inflammation regulator. Activation of NF $x \mathrm{~b}$ can also interfere with FXR and liver X receptor (LXR) signaling, which results in transcriptional suppression of bile and sterol transporters, finally culminating in cholestasis ${ }^{[20]}$. The above evidence indicates that hepatic macrophages, namely Kupffer cells (KCs), exert a pivotal role during the development of CLD.

Traditional Chinese medicines (TCMs) have been demonstrated to be an important source for potential drug discovery ${ }^{[21]}$. Tectorigenin (TEC), a plant isoflavone, has attracted much attention due to its multiple activities such as antiproliferation, antiinflammatory and antioxidant effects. We and others have demonstrated that TEC can inhibit macrophage activation (M1 polarity) in vivo and in vitro ${ }^{[22,23]}$. However, the underlying molecular mechanisms still require further investigation. It is noteworthy that TEC has been widely reported to provide protective functions in the liver ${ }^{[2-26]}$. This evidence prompted us to hypothesize that TEC could alleviate CLD by suppressing hepatic macrophage recruitment and activation. Thus, in the present study, we investigated whether TEC intervention could improve the development of CLD in the ANIT-induced and DDC-induced mouse models. We also investigated the molecular mechanism by which TEC regulates the polarization of bone marrow-derived macrophages (BMDMs) and primary mouse KCs.

\section{Results}

TEC alleviated ANIT-induced and DDC-induced experimental intrahepatic cholestasis

A previous study reported that ANIT and DDC treatment could cause intestinal permeability, leading to the translocation of bacteria or their products (LPS) from the leaky intestine into the liver ${ }^{[14]}$. Additionally, whilst murine experimental models may not fully represent human disease, ANIT and feeding with $0.1 \%$ DDC are well-accepted models of sclerosing cholangitis relevant to PSC ${ }^{[27,28]}$. Thus, the hepatoprotective effect of TEC was further confirmed in an experimental model of ANIT-induced and DDC-induced CLD.

The ANIT-induced mouse model exhibits increased cholestasis-serum markers and multiple large areas of necrosis, which can be significantly relieved by TEC treatment (Figure 1A and 1B). In line with these results, TEC inhibited ANIT-induced apoptotic cell death, as seen by fewer positive areas on TUNEL staining and reduced caspase-3 activity after TEC intervention (Figure 1C and 1D). Additionally, TEC alleviated ANITinduced inflammation as shown by a significant decrease in the recruitment of macrophages in the liver (Figure $1 \mathrm{E}$ and $1 \mathrm{~F}$ ). Consistent with this, our results showed that ANIT treatment significantly increased the content of IL-1 $\beta$ in mouse serum (Figure $1 G$ ) and the mRNA level of inflammatory factors in the liver (Figure 1H). As expected, TEC intervention reduced the serum level of IL-1 $\beta$ and the expression of hepatic inflammatory factors. 

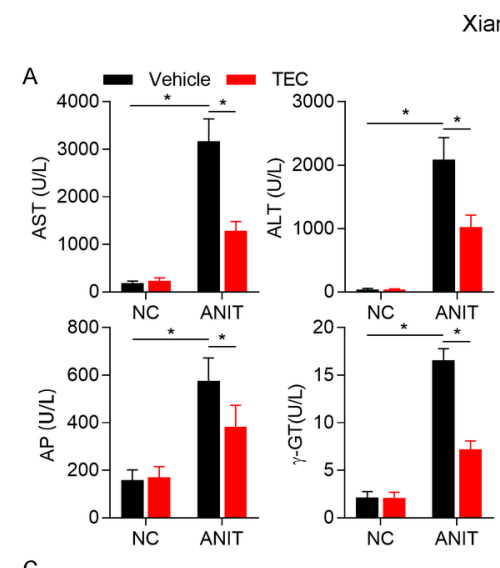

Xiang et al, Figure 1

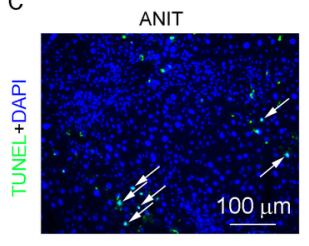

B
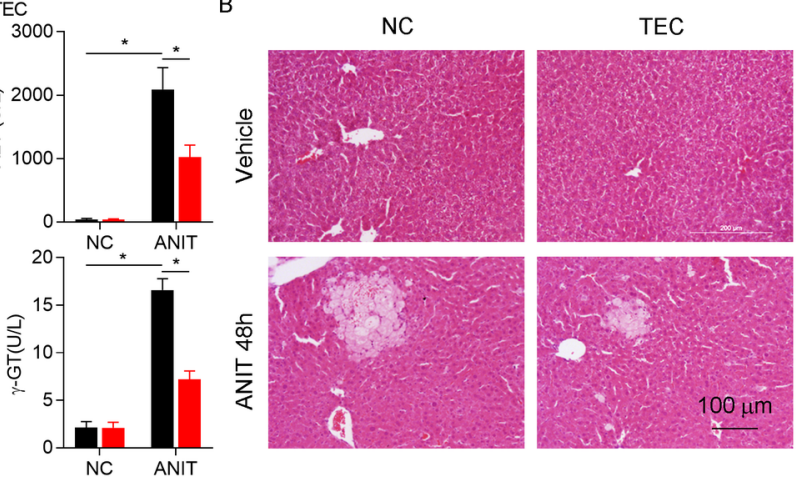

ANIT+TEC
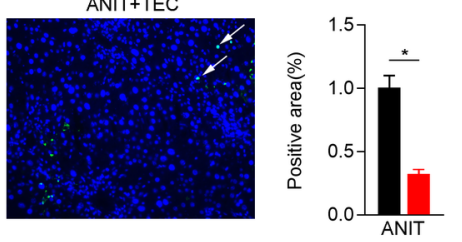

D
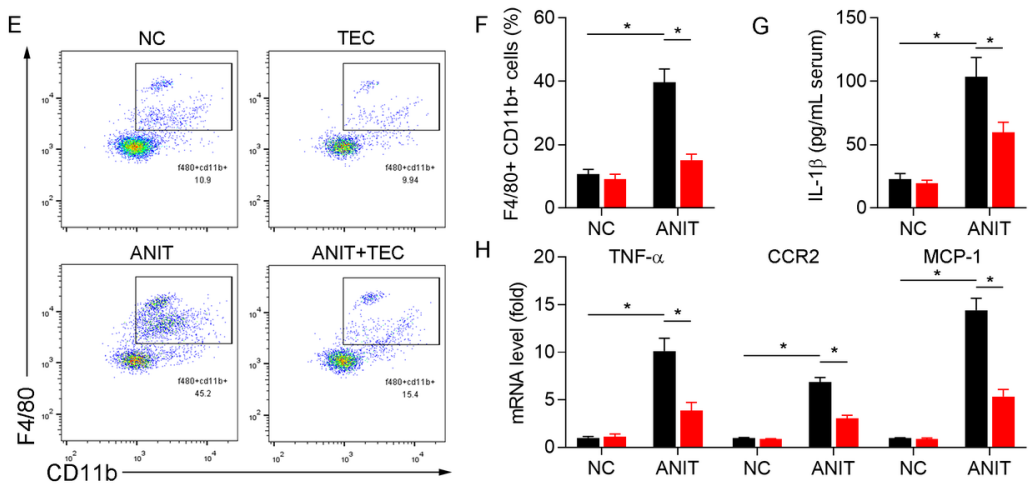

Figure 1. TEC alleviates ANIT-induced intrahepatic cholestasis.C57BL/6J mice were pre-treated with TEC for 3 days followed by ANIT treatment for $48 \mathrm{~h}$. (A) Serum level of AST, ALT, $\gamma$-GT, and $\mathrm{AP}$ were measured by ELISA kits, $\mathrm{n}=6$. (B) Representative images of hepatic HE staining were shown. (C) Representative images of hepatic TUNEL staining (left) and quantitative statistics (right) are shown in the figure. (D) The hepatic activity of caspase- 3 was determined by following the kit instructions, $\mathrm{n}=6$. (E-F) E: liver-isolated immune cells were stained for CD11b and F4/80 and analyzed by flow cytometry, representative dot plots are shown. F: further quantification of flow cytometry. (G) ELISA kits were used to detect inflammatory factors (IL-1 $\beta$ ) in serum, $n=6$. (H) The mRNA levels of TNF- $\alpha$, CCR2 and MCP-1 were measured by $\mathrm{qRT}-\mathrm{PCR}, \mathrm{n}=6 .{ }^{*} \mathrm{p}<0.05$. The data represent the mean $\pm \mathrm{SD}$.

Further experiments in mice fed with TEC in a diet containing $0.1 \%$ DDC for 2 weeks confirmed hepatocyte protection by TEC in cholestatic liver. DDC-fed mice showed marked liver injury compared to wild-type mice fed normal chow, as shown by increased AP, aspartate transaminase (AST), alanine transaminase (ALT) and $\gamma$-glutamyltransferase $(\gamma$-GT) (Figure $2 \mathrm{~A})$. The results of HE staining and Sirius red staining also supported these findings, as seen by decreased areas of tissue damage and collagen deposition in mouse liver after TEC intervention (Figure 2B and 2C). Flow cytometry analysis showed that the $0.1 \%$ DDC diet increased the recruitment of macrophages in the liver of mice (Figure 2D and $2 \mathrm{E}$ ). In accordance with this result, the mRNA level of inflammatory and profibrogenesis factors were also dramatically increased in $0.1 \%$ DDC-fed 
mice (Figure 2F and 2G). As expected, TEC significantly reduced hepatic macrophage recruitment, and the expression of inflammatory and profibrogenesis factors in the liver.

Overall, our results show that TEC treatment attenuated liver injury induced by ANIT or DDC.

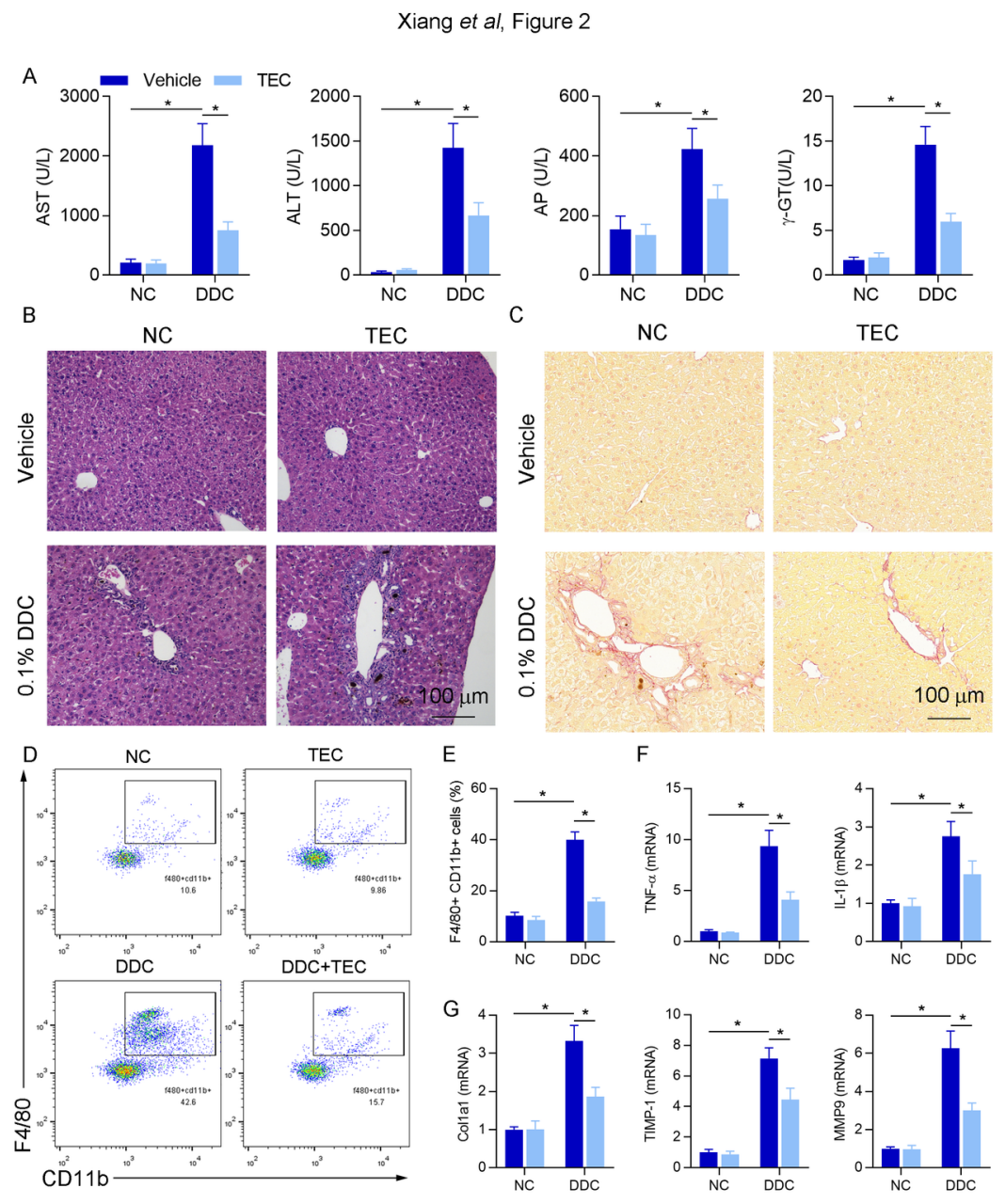

Figure 2. TEC protects mice from 0.1\% DDC diet-induced liver injury and inflammation. C57BL/6J mice were fed with $0.1 \%$ DDC and/or TEC for 1 week, and then sacrificed at the end of the experiment. (A) Serum level of AST, ALT, $\gamma$-GT, and AP were measured by ELISA kits, $n=6$. (B) Representative images of hepatic HE staining are shown. (C) Representative images of hepatic Sirius red staining are shown. (D-E) D: liver-isolated immune cells were stained for CD11b and F4/80 and analyzed by flow cytometry, representative dot plots are shown. E: further quantification of flow cytometry. (F-G) Hepatic mRNA levels of TNF- $\alpha$, IL- $1 \beta$, Col1 $\alpha 1$, TIMP-1 and MMP9 were measured by qRT-PCR, $\mathrm{n}=5$. $*_{\mathrm{p}}<0.05$. The data represent the mean $\pm \mathrm{SD}$.

TEC rescued bile metabolic dysfunction in mice with intrahepatic cholestasis induced by ANIT or DDC

We then undertook experiments to verify bile metabolism after TEC intervention in the CLD experimental model. Consistent with previous studies ${ }^{[29]}$, ANIT treatment alone resulted in the accumulation of bile acids in the liver and serum of mice, and reduced the content of bile acids in feces, which suggested that bile 
flow was mainly obstructed from the liver to the intestine (Figure 3A). CLD in mice was associated with reduced hepatic gene expression of canalicular transporters of bile salts (Bsep encoded by Abcb11), bilirubin (Mrp2, encoded by Abcc2), and phytosterols (the heterodimer sterolin1 and sterolin2, encoded by ABCG5 and ABCG8 ${ }^{[20,29,30]}$. Concomitant with biochemical cholestasis, mRNA expression of export transporters (Mrp2 and Bsep), phytosterol transporters (ABCG5 and ABCG8) and uptake transporters (Ntcp, Oatp) were significantly suppressed in mice with ANIT-induced CLD (Figure 3B). TEC significantly decreased the bile acid pool size in the liver and serum, and markedly increased the bile acid content in feces. Similarly, ANITinduced inhibition of transporters was reversed after TEC intervention. FXR is the master regulator of bile acids transporters (Bsep, Mrp2 and Ntcp), while LXR is an important regulator of ABCG5 and ABCG8 ${ }^{[20]}$. Therefore, western blotting analysis was performed, and the results showed that TEC intervention restored ANIT-induced decrease in FXR and LXR (Figure 3C).

Additionally, in $0.1 \%$ DDC-treated mice bile acids accumulated in the liver and serum, but decreased in feces (Figure 3D). Analysis of the expression of hepatic bile acid and phytosterol export transporters confirmed the expected reduction of the bile acid uptake transporters (Ntcp and Oatp), bile acid export transporters (Mrp2 and Bsep) and phytosterol export transporters (ABCG5 and ABCG8) after 0.1\% DDC treatment (Figure 3E). Notably, TEC significantly rescued the bile acid pool size in the liver, serum and feces. In line with this finding, TEC improved the expression of bile acid uptake transporters (Oatp and Ntcp), bile acid export transporters (Mrp2 and Bsep) and phytosterol export transporters (ABCG5 and ABCG8) in 0.1\% DDC fed mice. The expression of FXR and LXR was determined by western blot analysis, and the results showed that TEC restored DDC-induced downregulation of FXR and LXR in mouse liver (Figure 3F).

Taken together, these results showed that TEC attenuated ANIT-induced and DDC-induced bile metabolic dysfunction, and exerted a protective effect on CLD. 


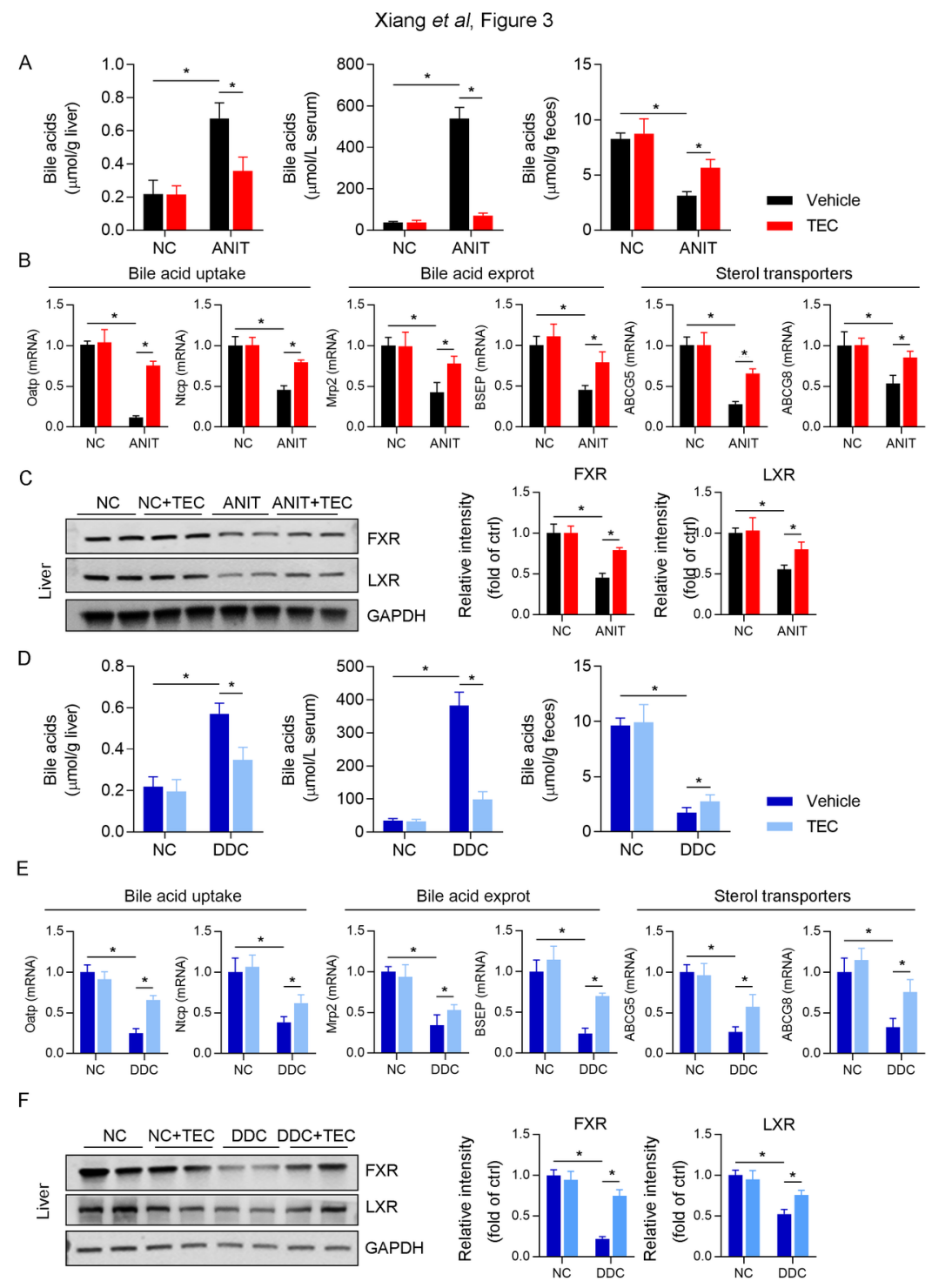

Figure 3. TEC restored ANIT and DDC-induced hepatic bile acid metabolic dysfunction. (AC) Liver, serum and feces samples were collected from ANIT and/or TEC treatment mice in Figure 1. A: bile acid pool size in the liver, serum and feces, $\mathrm{n}=6$, was determined by ELISA kits. B: bile acid uptake (Oatp, Ntcp), bile acid export (Mrp2, Bsep) and sterol transporters (ABCG5 and ABCG8) genes were determined by qRT-PCR, $n=5$. C: western blot analysis was performed to determine the protein level of FXR and LXR in mouse liver, and quantitative statistics are shown in the right panel, $\mathrm{n}=5$. (D-F) Liver, serum and feces samples were collected from DDC and/or TEC treated mice in Figure 2. D: bile acid pool size in the liver, serum and feces, $\mathrm{n}=5$, was determined by ELISA kits. E: bile acid uptake (Oatp, Ntcp), bile acid export (Mrp2, Bsep) and sterol transporters (ABCG5 and ABCG8) genes were determined by qRT-PCR, n=5. F: western blot analysis was performed to determine the protein level of FXR and LXR in mouse liver, and quantitative statistics are shown in the right panel, $\mathrm{n}=5 .{ }^{*} \mathrm{p}<0.05$. The data represent the mean $\pm \mathrm{SD}$.

TEC regulates macrophage activation dependent on PPAR $\gamma$ 
Previous studies have shown that TEC is a partial peroxisome proliferator-activated receptor- $\gamma$ (PPAR $\gamma$ ) agonist with an $\mathrm{IC}_{50}$ value of $13.3 \mu \mathrm{M}^{[31]}$. PPAR $\gamma$ activation inhibits the production of TNF- $\alpha$ and IL- $1 \beta$ in monocytes and macrophages, and promotes alternative macrophage activation ${ }^{[32]}$.

To confirm the regulation of TEC in KCs, we used primary KCs isolated from C57BL/6J mice. In vitro, TEC treatment inhibited LPS-induced activation of KCs, which proved that TEC markedly increased the M2 markers (IL-10, Arg-1, Retnla, CD163 and CD80) and decreased the M1 markers (TNF- $\alpha$, INF- $\gamma$, CCL2, CXCL1 and iNOS) (Figure 4A). Then, GW9662 (PPAR $\gamma$ inhibitor) was used to confirm that TEC regulation of KCs polarity was dependent on PPAR $\gamma$. Our results showed that TEC treatment did not further influence macrophage activation (TNF- $\alpha$, CXCL1, iNOS and CCL2) and macrophage alternative activation (IL-10, Arg-1, Retnla, CD206) when treated with GW9662 in the presence of LPS (Figure 4B). Analogical results were obtained by cotreatment with GW1929 (PPAR $\gamma$ agonist) and TEC (Figure S1A and S1B). Importantly, PPAR $\gamma$ expression deficiency dramatically suppressed TEC-mediated inhibition of macrophage activation in the presence of LPS (Figure 4C and 4D).

It is worth noting that BMDMs infiltrated the liver under CLD conditions. Thus, BMDMs were isolated from mice, and treated with TEC and/or ILPS. As expected, TEC increased the ratio of CD206/CD80, decreased M1 marker genes (TNF- $\alpha$, iNOS, IL-1 $\beta$ and CCL2) and upregulated the expression of M2 marker genes (IL-10, Arg-1, Retnla and CD163) (Figure S2A). BMDMs treated with shPPAR $\gamma$ and TEC supported our findings that TEC did not inhibit LPS-induced macrophage activation when PPAR $\gamma$ was knocked out (Figure S2B).

Accumulated evidence suggests that PPAR $\gamma$ attenuates inflammation by suppressing NF $\varkappa$ b activity. Mechanically, PPAR $\gamma$ is an E3 ligase that induces the degradation of NF $\varkappa b-p 65$ to terminate NF $\varkappa \mathrm{B}$ signaling pathway-elicited inflammation ${ }^{[33]}$. In light of this, we further determined whether TEC-regulated macrophage activation was dependent on the PPAR $/$ NF $\varkappa b$ pathway. BAY 11-7085 (NF $\varkappa b$ inhibitor) and/or TEC were used to treat KCs in the presence of LPS (Figure S1B). Our results showed that inhibition of NF $x$ b activity abolished the TEC-mediated regulation of KCs activation.

Taken together, these results indicate that TEC notably inhibited LPS-induced activation of macrophages. Moreover, TEC regulated macrophage activation which was dependent on activating PPAR $\gamma$. 


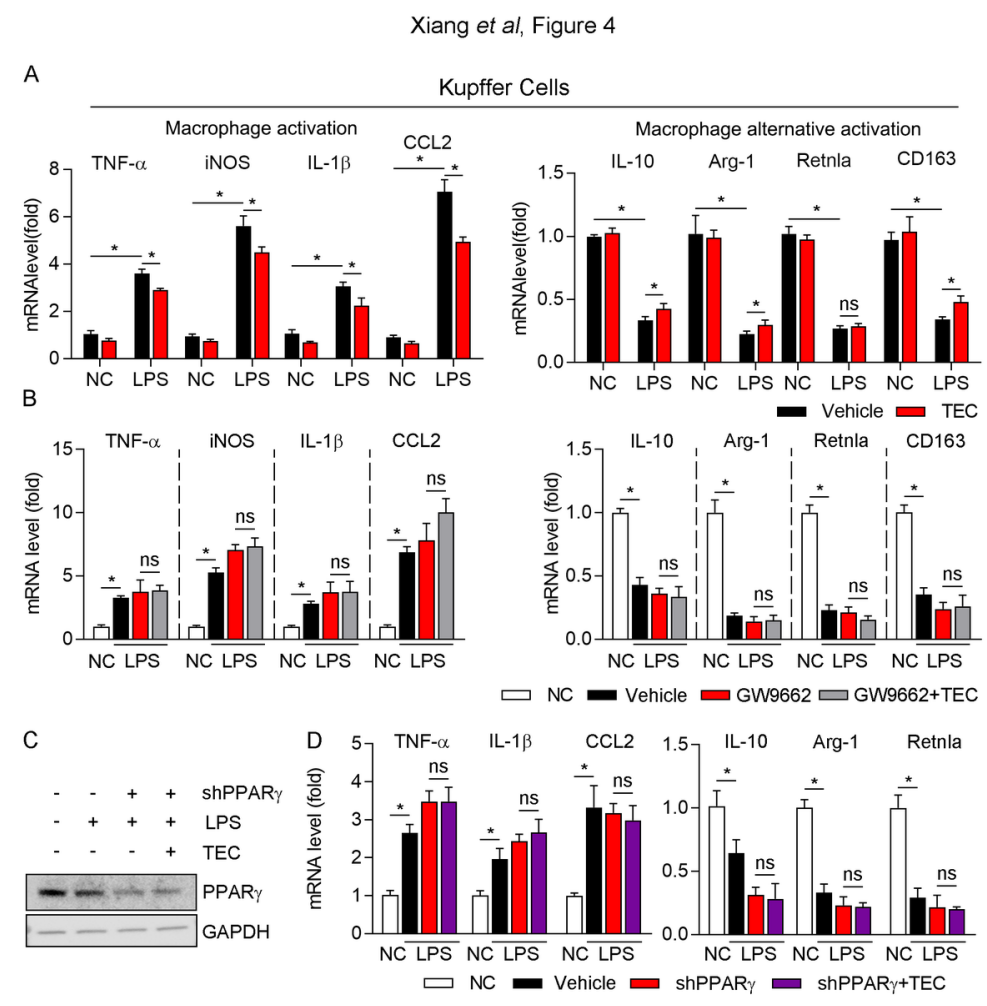

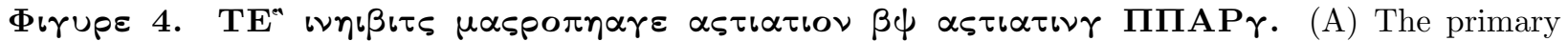
culture of KCs was treated with LPS $(0.1 \mu \mathrm{g} / \mathrm{mL})$ with or without TEC $(10 \mu \mathrm{M})$ for $24 \mathrm{~h}$. The mRNA levels of macrophage activation markers (TNF- $\alpha$, iNOS, IL- $1 \beta$ and CCL2) and macrophage alternative activation markers (IL-10, Arg-1, Retnla, CD206 and CD163) were measured by qRT-PCR, n=5. (B) The primary culture of KCs was treated with TEC and/or GW9662 (PPAR $\gamma$ antagonist, $10 \mu \mathrm{m}$ ) for $24 \mathrm{~h}$ in the presence of LPS. The mRNA level of TNF- $\alpha$, iNOS, IL-1 $\beta$, CCL2, IL-10, Arg-1, Retnla and CD163 was determined by qRT-PCR, $n=5$. (C-D) KCs treated with shPPAR $\gamma$, TEC and LPS as shown in the figure. C: representative images of western blot assay. D: the mRNA levels of TNF- $\alpha$, IL-1 $\beta$, CCL2, IL-10, Arg-1 and Retnla were detected by qRT-PCR, $\mathrm{n}=5 .{ }^{*} \mathrm{p}<0.05$. The data represent the mean $\pm \mathrm{SD}$.

\section{TEC directly improved the LPS-induced insults on FXR and LXR in hepatocytes}

The molecular basis for LPS-induced cholestasis has mainly been examined in hepatocytes and has been attributed to decreased expression of several transporters important for canalicular bile formation ${ }^{[34,35]}$. Mechanically, it was previously reported that NFxb-p65 can bind to FXR and LXR promoters, inhibit the expression of FXR and LXR, and lead to the decreased expression of bile transporters, resulting in the retention of bile acids and phytosterol. Phytosterol would then further inhibit the activity of $\operatorname{FXR}^{[36]}$. Therefore, we investigated whether TEC could directly suppress LPS-induced activation of NF $x$ b-p65 in hepatocytes. Primary hepatocytes were treated with LPS and/or TEC, and it was found that TEC reversed LPS-induced NF $x b-p 65$ activation, as shown by decreased phosphorylation of NF $x b-p 65$ and reduced NF $x$ bp65 binding to the FXR and LXR promoter (Figure 5A-C). In addition, TEC reversed the LPS-inhibited expression of FXR and LXR, as well as the expression of Bsep and ABCG5 (Figure 5D). To determine whether TEC regulates the expression and activity of FXR and LXR via PPAR $\gamma$, hepatocytes were treated with LPS, shPPAR $\gamma$ and TEC as indicated in Figure 5E. The results showed that PPAR $\gamma$ expression deficiency in hepatocytes blocked the regulatory effects of TEC on NF «b-p65 activity, the expression of FXR, Bsep, 
LXR and ABCG5 (Figure 5E and 5F). Furthermore, when NF «b-p65 was inhibited, TEC treatment could not further enhance the expression of FXR and LXR as well as FXR-regulated and LXR-regulated genes in the presence of LPS (Figure S3A-C). Finally, FXR or LXR deficiency abolished the influence of TEC on the expression of Mrp2, ABCG5 and ABCG8, but not Bsep (Figure S4A-B). Combined with the results of Bsep in Figure 5D and 5F, this suggested that TEC regulated the expression of Bsep, which was partly dependent on FXR and completely relied on PPAR $\gamma$.

TEC directly restored LPS-induced dysfunction of hepatocytes by activating PPAR $\gamma$.

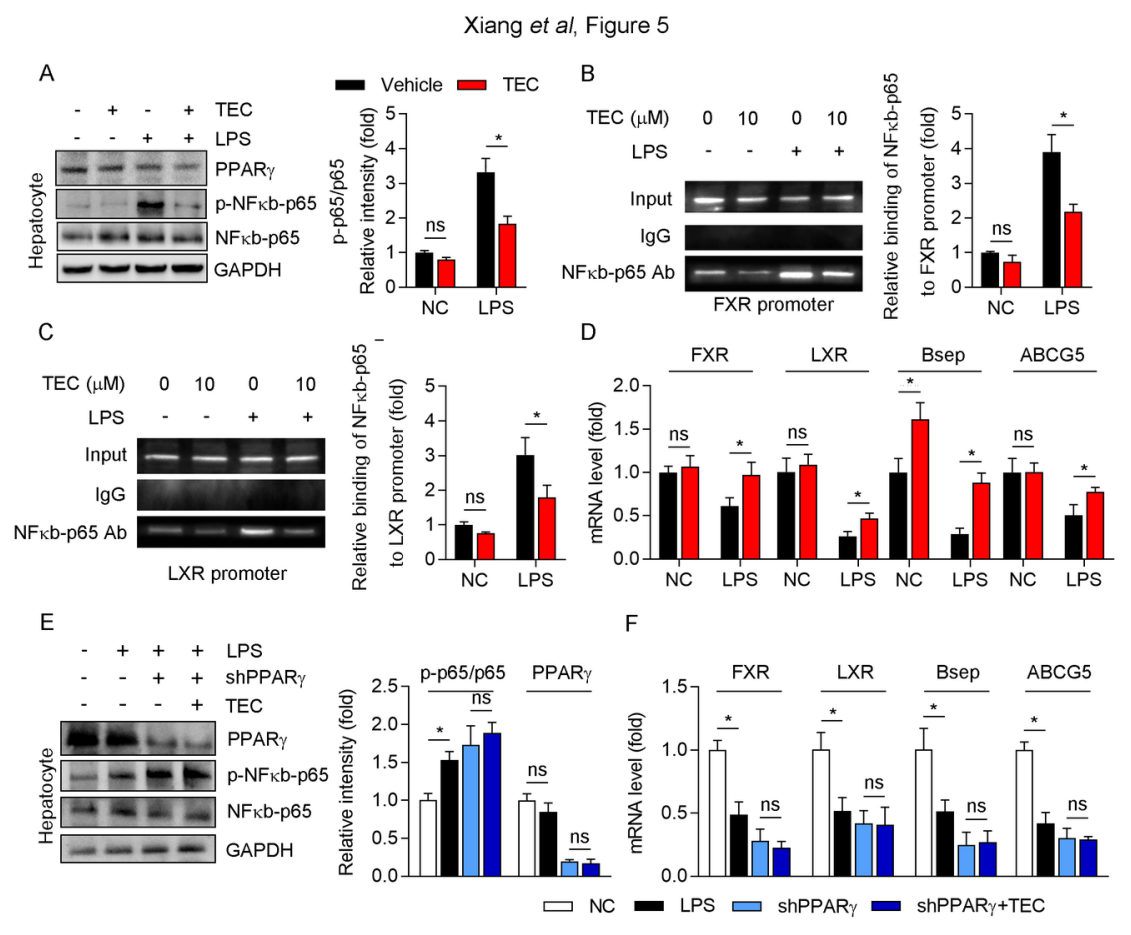

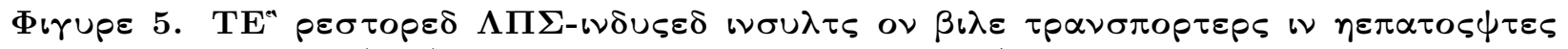

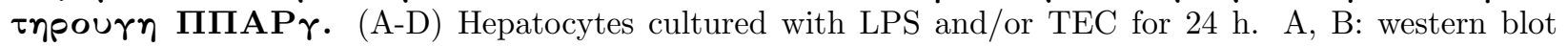
was used to detect the phosphorylation of NFxb-p65, n=5. B, C: Chromatin immunoprecipitation (ChIP) of hepatocytes with specific antibodies for NF $x b-p 65$ subunits binding to the promotor of mouse FXR (B) or the promotor of mouse LXR (C) represented as semiquantitative data (left) and quantitative PCR data with specific primers (right). D: the mRNA levels of FXR, LXR, Bsep and ABCG5 were determined by qRT-PCR, n=5. (E and F) Hepatocytes were transfected with shPPAR $\gamma$ and/or TEC in the presence of LPS. E: phosphorylation and protein level of NFxb-p65 was measured by western blot and quantitative data are shown on the right, $\mathrm{n}=5$. F: qRT-PCR was used to determine the mRNA expression of FXR, LXR, Bsep and ABCG5, $\mathrm{n}=5 .{ }^{*} \mathrm{p}<0.05$. The data represent the mean $\pm \mathrm{SD}$.

TEC promoted PPAR $\gamma$ binding to the Bsep promoter region and up-regulated its expression

In vitro studies have found that thiazolidinedione compounds (TZDs) can increase the expression of Bsep, but the exact molecular mechanism is still not fully understood ${ }^{[37]}$. Consistent with our current research results, TEC regulated the expression of Bsep independent of NFxb activity in hepatocytes (Figure 5D). Western blotting analysis showed that TEC increased the expression of Bsep, but not PPAR $\gamma$ in HepG2 cells (Figure 6A). When HepG2 cells were treated with TEC and GW1929 or TEC and GW9662, TEC did not further regulate the expression of Bsep compared with GW1929 or GW9662 treatment alone (Figure 6B). 
Further analysis revealed that TEC regulation of Bsep was completely dependent on PPAR $\gamma$, as PPAR $\gamma$ deficiency blocked the regulation of Bsep by TEC (Figure 6C). Importantly, the luciferase assay showed that TEC enhanced the transcriptional activity of PPAR $\gamma$ in a dose-dependent manner (Figure 6D).

Thus, we hypothesized that TEC increased the binding of PPAR $\curlyvee$ to the Bsep promoter and promoted its transcription. To gain further insight into the molecular mechanism of the regulation of Bsep by TEC, a series of Bsep promoter deletion mutants were cloned into the luciferase reporter system, and it was found that overexpression of PPAR $\gamma$ enhanced Bsep activity only in the Luc1 constructs, suggesting that the PPAR $\gamma$ binding site within the Bsep promoter was between the -2948 and -2235 base pairs (bp) (Figure 6E). Chromatin immunoprecipitation (ChIP) assay was used to confirm that PPAR $\gamma$ was recruited to the -2948 and $-2235 \mathrm{bp}$ (Figure $6 \mathrm{~F}$ ). The promoter analysis tool JASPAR predicted putative PPAR $\gamma$ binding motif sequences (-2560 bp to $-2541 \mathrm{bp}$ : ttggtccacagtgacctcca) based on the PPAR $\gamma$ consensus sequence outlined (Figure 6G). These results collectively indicated that TEC treatment increased the binding of PPAR $\gamma$ to the Bsep promoter region, thereby upregulating its expression.

\section{Xiang et al, Figure 6}
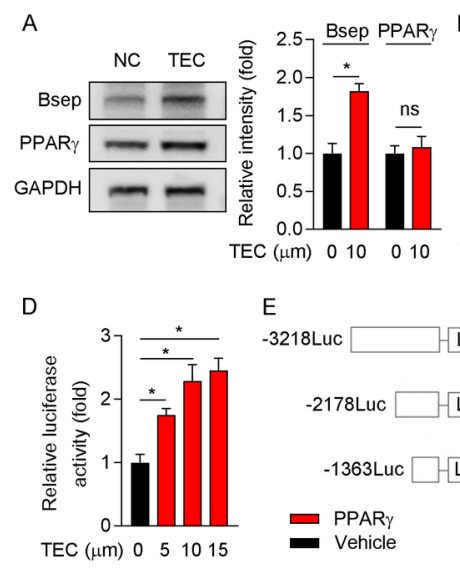

E
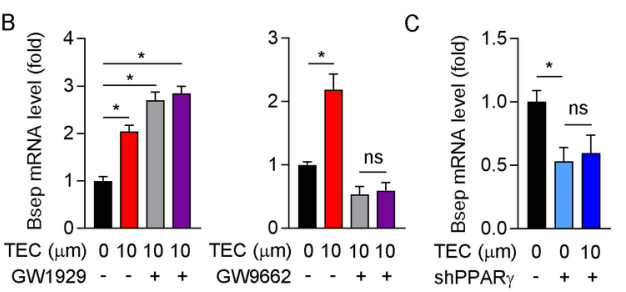

-3218Luc GW1929 -

G

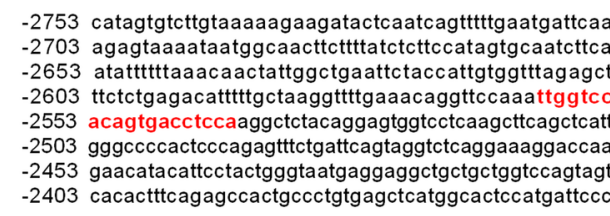

F
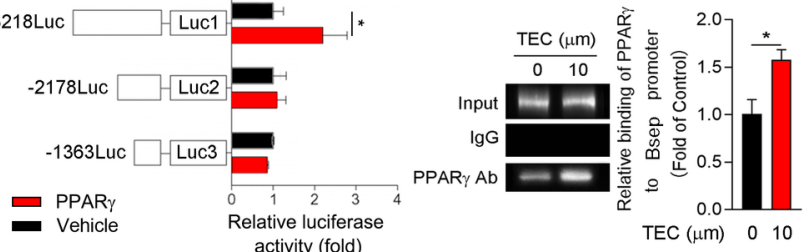
activity (fold) EC $(\mu \mathrm{m}) \quad 0 \quad 10$

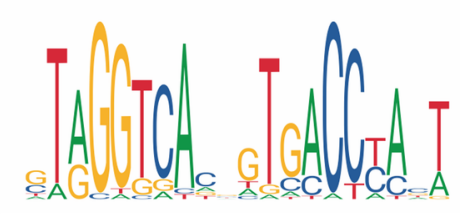

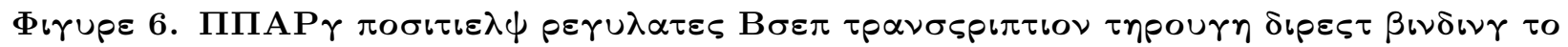

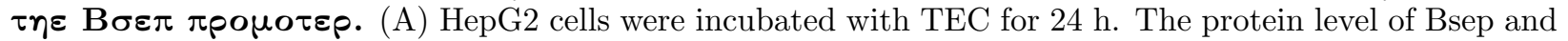
PPAR $\gamma$ were detected by western blotting, $n=5$. (B) HepG2 cells were incubated with TEC and/or GW1912 (GW9662) for $24 \mathrm{~h}$, and the mRNA level of Bsep was determined by qRT-PCR, n=5. (C) HepG2 cells were transfected with shPPAR $\curlyvee$ for $24 \mathrm{~h}$, then treated with TEC for another $24 \mathrm{~h}$, and the mRNA level of Bsep was determined by qRT-PCR, $\mathrm{n}=5$. (D) HepG2 cells were incubated with various concentrations of TEC for $24 \mathrm{~h}$, and PPAR $\gamma$ transcriptional activity was measured by luciferase assay, $\mathrm{n}=5$. (E) Luciferase reporter assays of a series of Bsep promoter fragments cloned into luciferase reporter gene (Luc1 to Luc3) with or without PPAR $\gamma$-transfection of HepG2 cells, n=5. (F) Chromatin immunoprecipitation (ChIP) assay-qPCR analysis in the region $-3218 \mathrm{bp} /-2178 \mathrm{bp}$ of the Bsep promoter in PPAR $\gamma$-transfected HepG2 cells with or without TEC treatment. IgG, immunoglobulin $\mathrm{G}, \mathrm{n}=5$. (G) Depiction of the predicted PPAR $\curlyvee$ binding sites at -2560 to -2541 base pairs (bp) in the human Bsep gene. ${ }^{*} \mathrm{p}<0.05$. The data represent the mean \pm SD. 
PPAR $r$ is essential for TEC to alleviate ANIT-induced experimental intrahepatic cholestasis

To further confirm the physiological roles of PPAR $\gamma$ in TEC-mediated hepatic protection in vivo, AdshPPAR $r$ was injected into C57BL/6J mice via the tail vein. Next, the mice were treated with ANIT and/or TEC as described in the experimental method. As expected, TEC did not exert hepatic protection in ANITinduced CLD of PPAR $\gamma$ deficient mice, which was supported by the fact that there was no obvious difference in AST, ALT, AP and necrosis area after TEC treatment in PPAR $\gamma$-deficient mice (Figure 7A and 7B). Quantification of the bile acid pool size showed that there was no significant influence on the content of bile acid in the liver, serum and feces of PPAR $\gamma$-deficient mice after TEC treatment (Figure 7C). ANIT treatment alone markedly promoted macrophage recruitment compared with $\mathrm{NC}$, as evidenced by a significant increase in F4/80 mRNA level in the liver (Figure 7D). The mRNA levels of proinflammatory factors (Figure 7E) and profibrogenesis factors (Figure $7 \mathrm{~F}$ ) also supported these findings. PPAR $\gamma$ deficiency aggravated inflammation in mouse liver, as shown by increased F4/80, proinflammatory factors and profibrogenesis factors compared with ANIT treatment alone, while TEC did not significantly decrease these genes in PPAR $\gamma$ deficient mice. Furthermore, the expression of FXR and LXR in mouse liver was analyzed, and the results showed that TEC did not enhance the expression of FXR, LXR and bile transporters in PPAR $\gamma$-deficient mice (Figure $7 \mathrm{G}$ and $7 \mathrm{H})$. Taken together, the lack of PPAR $\gamma$ expression abolished hepatic protection by TEC. 


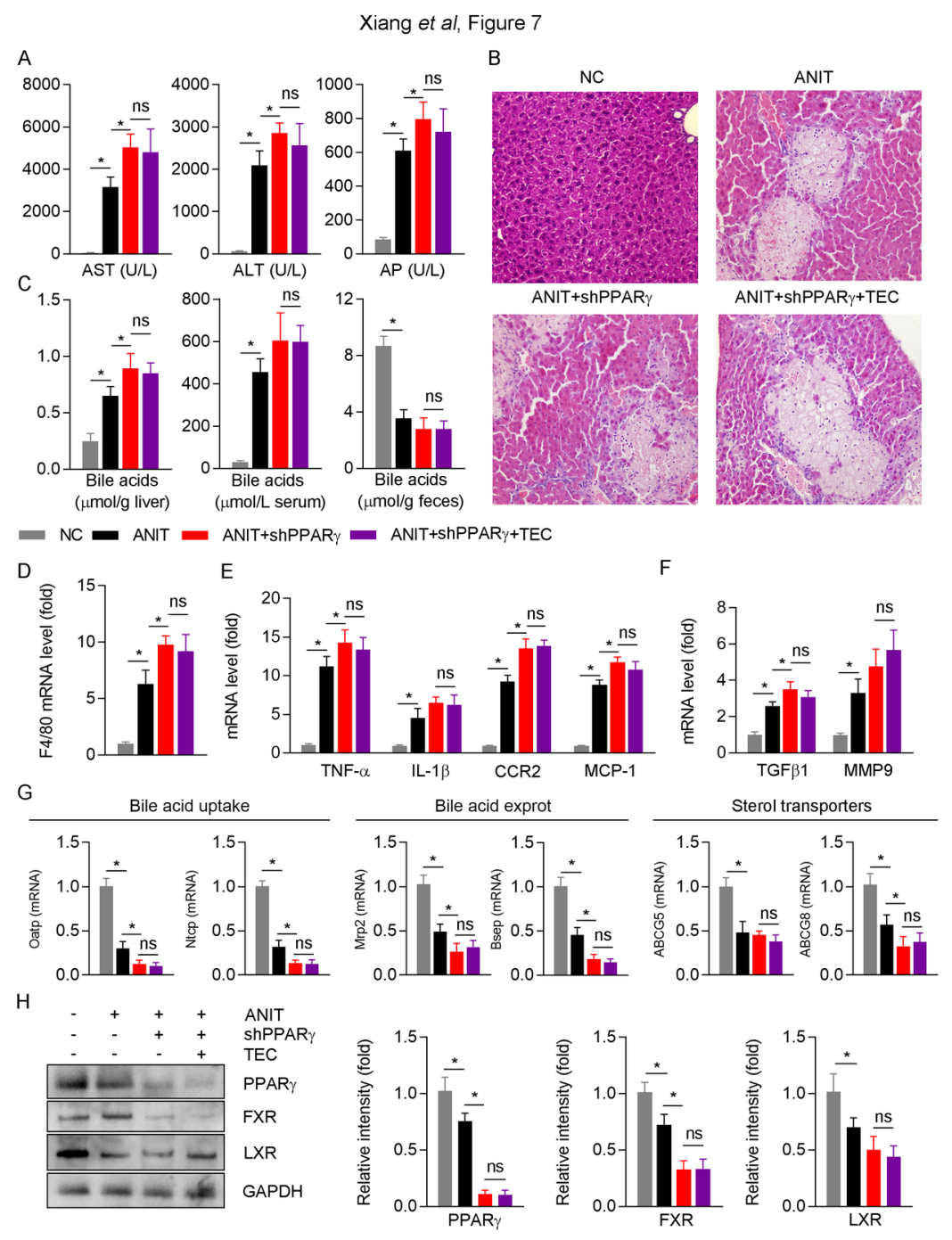

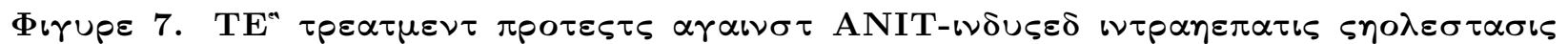

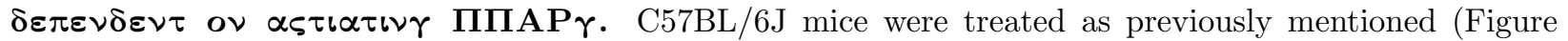
1). (A) The serum levels of aspartate transaminase (AST), alanine transaminase (ALT) and AP were determined by an ELISA kit, $n=6$. (B) Representative sections stained with HE were used to estimate the area of necrosis. (C) Bile acid pool size was determined by ELISA kits in the liver, serum and feces, $\mathrm{n}=6$. (D-F) The mRNA level of macrophage recruitment (F4/80), inflammatory factors (TNF- $\alpha$, IL-1 $\beta$, MCP-1 and CCR2) and fibrosis-related genes (TGF $\beta 1$, MMP9, TIMP-1 and col1 $\alpha 1$ ) were measured by qRT-PCR, $\mathrm{n}=6$. (G) qRT-PCR analysis was used to determine the mRNA level of Oatp, Ntcp, Mrp2, Bsep, ABCG5 and ABCG8, $n=6$. $(\mathrm{H})$ Western blot analysis was used to confirm the expression of PPAR $\gamma, \mathrm{FXR}$ and LXR, $\mathrm{n}=5 .{ }^{*} \mathrm{p}<0.05$. The data represent the mean $\pm \mathrm{SD}$.

\section{Discussion}

Various studies have demonstrated that PPAR $\gamma$ agonists have potential in the treatment of CLD. Immune modulation by PPAR $\gamma$ ligands may be of therapeutic benefit in reducing biliary inflammation in PBC ${ }^{[38]}$. In addition, PPAR $\gamma$ inhibits the transcriptional activation of inflammatory response genes and represses 
cellular toll-like receptor signaling in inflammatory cells as well as in cholangiocytes ${ }^{[39]}$. Importantly, recent studies have shown that rosiglitazone improve intrahepatic cholestasis and cholestasis-associated dyslipidemia induced by ANIT ${ }^{29]}$. These data indicated that PPAR $\gamma$ activation may be an effective strategy for the treatment of CLD, especially for the improvement of liver fibrosis and inflammation, thereby limiting disease progression. Despite this, troglitazone, a PPAR $\gamma$ ligand, was withdrawn from the market due to hepatotoxicity and no experimental or clinical data on other glitazones are available ${ }^{[40]}$. Although this evidence suggests the feasibility of PPAR $\Upsilon$ agonists as a therapeutic strategy for CLD, new agonists still need to be developed.

In this study, we demonstrated that TEC, a partial PPAR $\gamma$ agonist ${ }^{[31]}$, can alleviate cholestasis in an experimental mouse model (Figure 1 and 2) without obvious side effects. Importantly, we confirmed that TEC alleviation of ANIT-induced liver injury was dependent on PPAR $\gamma$ to reduce the recruitment and activation of macrophages and enhance bile transporter expression in hepatocytes (Figure 7). Taken together, we found that TEC may be a potential therapeutic strategy for the treatment of CLD as a PPAR $\gamma$ agonist.

It was shown in vitro that TEC inhibited LPS-induced macrophage activation as well as LPS-induced hepatocyte dysfunction via the PPAR $\Upsilon / \mathrm{NF} x \mathrm{~b}$ pathway (Figure 4 and 5). In accordance with our findings, multiple studies have suggested that activation of NF $x$ b plays an important role in the development of $\mathrm{CLD}^{[20,41]}$. Genetic or pharmacological inhibition of NFxb prevented cholestasis-induced liver damage in various experimental mice $\left.{ }^{[2,}, 43\right]$. However, inhibition of NF $x$ b was found to lead to an increase in hepatocyte apoptosis after bile duct ligation (BDL ${ }^{[44]}$. Additionally, IKK1 and IKK2 are I $\chi$ B kinases which are important for $\mathrm{NF} \chi \mathrm{b}$ activation and genetic ablation of $\mathrm{I} \chi \mathrm{B}$ kinases could lead to inflammatory damage to portal bile ducts ${ }^{[45]}$. Therefore, appropriate targets or the identification of drugs that either exert only a moderate effect on NF $x$ b activity or that can be specifically delivered to nonparenchymal cells are essential to avoid the increase in liver injury associated with complete NFxb blockade in hepatocytes ${ }^{[46]}$.

Interestingly, PPAR $\gamma$ is highly expressed in macrophages where it has an important role in immune modulation, while PPAR $\gamma$ expression is relatively low in hepatocytes under normal physiological conditions ${ }^{[47,48]}$. Our results showed that TEC treatment almost blocked the phosphorylation of NFxb-p65 induced by LPS in KCs (Figure S1C) and BMDMs (Figure S2D). The phosphorylation of NF $x$ b-p65 was still 1.78-fold higher than that in control hepatocytes after TEC treatment in the presence of LPS (Figure 5A). An in vivo study also supported these findings, where TEC decreased rather than increased the apoptosis of hepatocytes in ANIT-induced model mice, as shown by the down-regulation of the positive area of TUNEL staining and reduced caspase-3 activity (Figure $1 \mathrm{C}$ and D). Our results suggest that TEC had a much stronger inhibitory effect on NFxb in macrophages compared with hepatocytes which could be attributed to the different levels of PPAR $\gamma$ expression in KCs and hepatocytes. Additionally, NF $x \mathrm{~b}$ inhibitor (BAY 11-7085) treatment at the same dose as TEC markedly decreased the phosphorylation of NF $x b-p 65$ induced by LPS in hepatocytes, to an even lower level than that in the control group (Figure S3A). Collectively, this dose of TEC had a stronger inhibitory effect on NFxb activation in macrophages compared with hepatocytes, and avoided liver injury induced by complete NFxb blockade in hepatocytes.

Bsep, encoded by the gene ABCB11, is a member of the adenosine triphosphate (ATP)-binding cassette (ABC) transporters. It is mainly expressed on hepatocyte canalicular membranes and is basically responsible for the secretion of bile acids, and it deficiency may result in progressive familial intrahepatic cholestasis type $2^{[49]}$. De novo or retargeted canalicular expression of Bsep has been confirmed to play an important role in bile acid canalicular export in the treatment of cholestasis ${ }^{[50,51]}$. Previous studies have shown that troglitazone can induce intrahepatic cholestasis by increasing serum bile salt concentrations and inhibiting Bsep expression in rat liver ${ }^{[40,52]}$. In contrast, another study showed that troglitazone, but not rosiglitazone or pioglitazone, regulated the expression of the FXR target gene Bsep ${ }^{[37]}$. In summary, this evidence could support further investigation of the relationship between Bsep and TEC. According to our findings, TEC promoted the binding of PPAR $\gamma$ and Bsep promoter regions and promoted their expression (Figure 5). As TEC directly increased the expression of Bsep, this may be another molecular mechanism of TEC in the treatment of CLD. 
Although we found that TEC (50 mpk) significantly alleviated liver injury in ANIT and DDC-induced CLD without significant side effects, additional cholestatic models and different doses are still needed to verify the efficacy and toxicity of TEC. In conclusion, we have demonstrated that TEC exert liver protection in a PPAR $\gamma$-dependent manner, which in turn inhibit macrophage activation and hepatocyte dysfunction through restrain NF $x b$ activation as well as enhance Bsep expression, thus alleviated intrahepatic cholestasis.

\section{Materials and methods}

\section{Reagents}

Antibodies for FXR (Cat\#: ab129089), LXR (Cat\#: ab176323), GAPDH (Cat\#: ab181602), PPAR (Cat\#: ab178860), p-NFxb/P65 (Cat\#: ab76302), NFxb/P65 (Cat\#: ab16502), and Bsep (Cat\#: ab155421) were obtained from Abcam (Cvambridge, MA). Reagents of Tectorigenin (TEC) (Cat\#: HY-N0792), GW9662 (Cat\#: HY-16578), GW1929 (Cat\#: HY-15655), BAY11-7085 (Cat\#: HY-10257) were obtained from MedChemExpress (Monmouth Junction, NJ), and Lipopolysaccharides (LPS) (Cat\#: L2630) and alphanaphtylisocyanate (ANIT) were obtained from Sigma-Aldrich (Shanghai, China). Mouse AST ELISA Kit (Aspartate Aminotransferase) (Cat\#: ab263882), ALT Assay Kit (Cat\#: ab241035), Caspase-3 Activity Assay Kit (Cat\#: ab252897), AP Assay Kit (Cat\#: ab267583), and Gamma Glutamyl Transferase $(\gamma$ GT) Assay Kit (Cat\#: ab241029) were obtained from Abcam (Cambridge, MA). Mouse IL-1ß ELISA KIT (Cat\#: SEKM-0002) was obtained from Solarbio \& Technology Co, Ltd (Beijing, China). Fluorescein (FITC) Tunel Cell Apoptosis Detection Kit (Cat\#: G1501), Sirius Red dyestuff (Cat\#:G1018), and HE dyestuff (Cat\#: G1005) were obtained from servicebio (Wuhan, China). FXR shRNA(m) (Cat\#: sc-155894), LXR $\alpha$ shRNA(m) (Cat\#: sc-38829), PPAR $\gamma$ shRNA(h) (Cat\#: sc-29455-V), and PPAR $\gamma$ shRNA(m) (Cat\#: sc29456) were obtained from Santa Cruz Biotechnology (Santa Cruz, CA). All other reagents were obtained from Sigma-Aldrich except where indicated.

\section{In vivo studies with animals}

All animal care and experimental protocols for in vivo studies conformed to the Guide for the Care and Use of Laboratory Animals published by the NIH (NIH publication no. 85-23, revised 1996) and approved by the Ethics Committee of the Second Clinical Medical College of Jinan University, Shenzhen People's Hospital. C57BL/6J wild type mice ( 8 weeks old) were purchased from the Animal Center of Nanjing University (Nanjing, Jiangsu, China). These mice were maintained at the Animal Center of Shenzhen People's Hospital with free access to food and water. Based on the previous study, the dose of TEC used to mice was converted as $75 \mathrm{mg} /$ day $/ \mathrm{kg}$ body weight (mpk). Cholestasis was induced was induced in C57BL/6J mice by administration of ANIT for $48 \mathrm{hr}$ by oral gavage or fed with a diet containing 1\% 3,5-diethoxycarbonyl1,4-dihydrocollidine (DDC) for 2 weeks. C57BL/6J mice were treated either with vehicle (corn oil) or ANIT $(100 \mathrm{mg} / \mathrm{kg})$ by oral gavage, while a subset of C57BL/6J mice were treated with TEC $(75 \mathrm{mpk})$ for 5 days and another subset of C57BL/6J mice treated with TEC by oral gavage 3 days before, at the time of the administration of ANIT, and 24h after. During the DDC feeding, TEC (75 mpk) was administered daily after DDC feeding for one week. All ANIT treated animals were sacrificed $48 \mathrm{hr}$ after gavage, while all $1 \%$ DDC treated mice 2 weeks after feeding. For constructed PPAR $\gamma$ knockdown mice, mice were injected i.v. through the tail vein with control adenovirus expressing shorthairpin (sh)RNA against luciferase (AdshCtrl) or adenovirus expressing shRNA against PPAR $\gamma(\mathrm{Ad}-\operatorname{shPPAR} \gamma)\left(0.5-1.5 \times 10^{9}\right.$ active viral particles in $200 \mu \mathrm{l}$ saline [ $54 \mathrm{mmol} / \mathrm{l} \mathrm{NaCl}]$ ). At 2 weeks after infection, mice performed experiments as mentioned

before. Extraction and determination of total bile acids (TBA) in mouse liver and intestine was performed as previously reported ${ }^{[29]}$.

\section{Cell Culture}

Primary KCs were prepared from the livers of 8- to 9-week-old male C57BL/6 mice and cultured in 10\% FBS RPMI 1640 medium .A single-cell suspension form livers was prepared as described previously ${ }^{[53]}$. BMDMs were isolated from femoral and tibia bone marrow and then cultured in RPMI1640 medium(10\%FBS) with $100 \mathrm{ng} / \mathrm{mL}$ M-CSF (Peprotech) for 5 days to fully differentiate macrophages. Primary mouse hepatocytes were obtained from the livers of male $\mathrm{C} 57 \mathrm{BL} / 6 \mathrm{~J}$ mice ( 8 weeks of age) and cultured as previously reported ${ }^{[54]}$. 
HepG2 cells, a human hepatic cell line, were purchased from ATCC (Manassas, VA, USA, RRID: CVCL 0027) and cultured in DMEM medium containing $10 \%$ fetal bovine serum (FBS), $50 \mathrm{mg} / \mathrm{mL}$ penicillin/streptomycin and $2 \mathrm{mmol} / \mathrm{L}$ glutamine. Cells ( $~ 90 \%$ confluence) received treatment in serum-free medium.

Quantitative real-time polymerase chain reaction (qRT-PCR), Western blotting, ChIP and ELISA

qRT-PCR was performed using the ABI StepOnePlus ${ }^{\mathrm{TM}}$ Real-time PCR system (Applied Biosystem) with specific primers (Table 1 ). The relative mRNA level of target genes was analyzed using the equation $2-\Delta \mathrm{Ct}$ $(\Delta \mathrm{Ct}=\mathrm{Ct}$ of the target gene $-\mathrm{Ct}$ of $\beta$-actin) and normalized using the level detected in the control group as 1. Western blotting experiments were performed using antibodies for FXR, LXR, and PPAR $\gamma$ etc., as described previously ${ }^{[22]}$. ChIP was performed as described ${ }^{[21]}$. ChIP was done using specifc antibodies for NF- $\chi \mathrm{B}$ p65 (Abcam, cat\# ab16502) or $\operatorname{PPAR} \gamma(\mathrm{Cat} \#:$ ab178860), and amplification of promoter sequences from the Nr1h3 (LXR $\alpha)$, Nr1h4 (FXR) and Bsep genes using specific primer sets and subsequent PCR; After elution and purification, the chromatin DNA was subjected to real time PCR analysis with primers, LXR $\alpha$ : forward: 5'- aaggaagctcaggcacaaaa -3' and reverse: 5'- gaggctgtgcttgtgaaaca -3', FXR: forward: 5'ccactggagatccaaaagga -3' and reverse: 5'- aatctatgcaaagcgctggt -3', Bsep primer sequences: forward: 5'tccaaattggtccacagtga -3' and reverse: 5'- agcagcagcctcctcattac -3'. The levels of level of AST, ALT, $\gamma$ GT, and AP etc., were measured using a commercial ELISA kit (Abcam) according to the manufacturer's protocol.

Table 1. The sequences of primers for qRT-PCR analysis

\begin{tabular}{|c|c|c|}
\hline Gene & Forward & Backward \\
\hline$\mu T N \Phi-a$ & GACGTGGAACTGGCAGAAGAG & TTGGTGGTTTGTGAGTGTGA \\
\hline$m C C R 2$ & ATCCACGGCATACTATCAACATC & CAAGGCTCACCATCATCGTAG \\
\hline$m M C P-1$ & TTAAAAACCTGGATCGGAACCAA & GCATTAGCTTCAGATTTACGG \\
\hline$m$ Col1a1 & GCTCCTCTTAGGGGCCACT & CCACGTCTCACCATTGGGG \\
\hline$m T I M P-1$ & GCAACTCGGACCTGGTCATAA & CGGCCCGTGATGAGAAACT \\
\hline$m M M P 9$ & CTGGACAGCCAGACACTAAAG & CTCGCGGCAAGTCTTCAGAG \\
\hline mOatp & GGGAACATGCTTCGTGGGATA & GGAGTTATGCGGACACTTCTC \\
\hline$m N t c p$ & CAAACCTCAGAAGGACCAAACA & GTAGGAGGATTATTCCCGTTG \\
\hline mMrp2 & GTGTGGATTCCCTTGGGCTTT & CACAACGAACACCTGCTTGG \\
\hline mBsep hBsep & TCTGACTCAGTGATTCTTCGCA GCCGCAGCTCGTCAGATAC & CCCATAAACATCAGCCAGTTG \\
\hline$m A B C G 5$ & AGGGCCTCACATCAACAGAG & GCTGACGCTGTAGGACACAT \\
\hline$m A B C G 8$ & CTGTGGAATGGGACTGTACTTC & GTTGGACTGACCACTGTAGG \\
\hline $\operatorname{miNOS}$ & GTTCTCAGCCCAACAATACAAGA & GTGGACGGGTCGATGTCAC \\
\hline$\mu I \Lambda-1 \beta$ & GCAACTGTTCCTGAACTCAACT & ATCTTTTGGGGTCCGTCAACI \\
\hline$m C C L 2$ & TTAAAAACCTGGATCGGAACCAA & GCATTAGCTTCAGATTTACGG \\
\hline mIL10 & GCTCTTACTGACTGGCATGAG & CGCAGCTCTAGGAGCATGTG \\
\hline mArg1 & CTCCAAGCCAAAGTCCTTAGAG & AGGAGCTGTCATTAGGGACA] \\
\hline mRetnla & CCAATCCAGCTAACTATCCСТCC & ACCCAGTAGCAGTCATCCCA \\
\hline$m C D 206$ & CTCTGTTCAGCTATTGGACGC & CGGAATTTCTGGGATTCAGC \\
\hline$m C D 163$ & ATGGGTGGACACAGAATGGTT & CAGGAGCGTTAGTGACAGCAC \\
\hline$m F X R$ & GCTTGATGTGCTACAAAAGCTG & CGTGGTGATGGTTGAATGTC \\
\hline$m L X R$ & ATGTCTTCCCCCACAAGTTCT & GACCACGATGTAGGCAGAGC \\
\hline$m F 4 / 80$ & TGACTCACCTTGTGGTCCTAA & CTTCCCAGAATCCAGTCTTTC \\
\hline$\mu Т Г \Phi \beta 1$ & CTCCCGTGGCTTCTAGTGC & GCCTTAGTTTGGACAGGATCI \\
\hline
\end{tabular}

\section{Flow cytometry}

KCs were incubated with anti-CD16/CD32 (BD 553141) FC receptor for 5 min at RT. And then cells were 
labeled with the following antibody at indicate dilutions. CD45-APC cy7(BD 557659),CD11b-FITC(BD 557391),F4/80-PE(BD 565410), after the 30 minute incubationat 4. KCs were washed and fixed in stain buffer FBS (BD 554656). Finally, analyses were performed on a Beckman DXFLEX Flow Cytometer.

\section{Dual luciferase reporter gene assay}

The full-length mouse Bsep gene was amplified by PCR from HepG2 cDNA. The mouse Bsep gene promoter (-3218 to -198 bp) was amplified by PCR using mouse genomic DNA and inserted into a pGL3-basic luciferase reporter vector (-3218 Luc). A series of 5' truncated constructs of the Bsep gene promoter (-3218 Luc, -2178 Luc, -1363 Luc) were prepared by PCR using -3218 Luc as a template. All constructs were confirmed by DNA sequencing analysis. For dual luciferase reporter gene assays, HepG2 were transfected with corresponding plasmids, as well as renilla luciferase. Firefly and renilla luciferase activities were then measured by a dual-luciferase reporter gene system (Promega, Madison, WI, USA).

\section{Histological analysis}

The mice tissues were preserved with $4 \%$ paraformaldehyde solution, dehydrated and embedded in paraffin. The sections $(4 \mu \mathrm{m})$ were used for histological analysis. HE, Sirus red and TUNEL staining performed as standard instructions. Positive cells were morphometrically quantified with image processing software (ImageJ).

\section{Data analysis}

All the data were generated from at least 3 independent experiments. All the raw data were initially subjected to a normal distribution anal analysis with SPSS software (1-sample K-S of nonparametric test). The data in normal distribution were conducted the parametric statistic, post hoc test of 1-way analysis of variance.

\section{Abbreviations}

ALT, alanine transaminase; ANIT, $\alpha$-naphthylisothiocyanate; AP, alkaline phosphatase;AST, aspartate transaminase; ATP, adenosine triphosphate ; BDL, bile duct ligation; BMDMs, bone marrow-derived macrophages; Bsep, bile salt export pump; CCR-2, C-C chemokine receptor type-2; CLD, cholestatic liver disease; DDC, 3,5-diethoxycarbonyl-1,4-dihydrocollidine; FXR, farnesoid X receptor; $\gamma$-GT, $\gamma$ glutamyltransferase; IL-1 $\beta$, interleukin 1 beta; KCs, Kupffer cells; LPS, lipopolysaccharide; LXR, liver $\mathrm{X}$ receptor; MCP, monocyte chemoattractant protein; NF $x \mathrm{~b}$, nuclear factor $\varkappa \mathrm{B}$; OCA, obeticholic acid; $\mathrm{PBC}$, primary biliary cholangitis; PPAR $\gamma$, peroxisome proliferator-activated receptor- $\gamma$ PSC, primary sclerosing cholangitis; TBA, total bile acids; TCMs, traditional chinese medicines; TEC, tectorigenin; TZDs, thiazolidinedione compounds; UDCA, ursodeoxycholic acid.

\section{Acknowledgments}

This work was supported by grants from Natural Science Foundation of Tianjin (19JCQNJC12600); Research project of Tianjin education commission (2019KJ044).

\section{Contributions}

JX, GY and CM contributed equally to this work. JX and CM performed in vivo experiments; GY, HW and LJ completed molecular biological experiments and biochemical assays; LK, ZL, LW and XT edited the manuscript; SY designed experiments, interpreted results and wrote the manuscript.

\#: These authors make equal contribution to this work.

\section{Competing interests}

All the authors state no conflict of interest.

\section{References}

[1] Dyson JK, et al., Novel Therapeutic Targets in Primary Biliary Cirrhosis. Nature reviews. Gastroenterology 83 hepatology, 2015. 12 (3) 147-158. 
[2] Easl Clinical Practice Guidelines: The Diagnosis and Management of Patients with Primary Biliary Cholangitis. Journal of hepatology, 2017. 67 (1) 145-172.

[3] Beuers U, et al., Changing Nomenclature for Pbc: From 'Cirrhosis' to 'Cholangitis'. Hepatology (Baltimore, Md.) , 2015. 62 (5) 1620-1622.

[4] Hirschfield GM, et al., Efficacy of Obeticholic Acid in Patients with Primary Biliary Cirrhosis and Inadequate Response to Ursodeoxycholic Acid.Gastroenterology , 2015. 148 (4) 751-761.e758.

[5] Nevens F, et al., A Placebo-Controlled Trial of Obeticholic Acid in Primary Biliary Cholangitis. The New England journal of medicine, 2016. 375 (7) 631-643.

[6] Yokoda RT, et al., Primary Biliary Cholangitis and Primary Sclerosing Cholangitis. The American journal of gastroenterology , 2019. 114 (10) 1593-1605.

[7] Quraishi MN, et al., The Gut-Adherent Microbiota of Psc-Ibd Is Distinct to That of Ibd.Gut , 2017. 66 (2) 386-388.

[8] Tang R, et al., Gut Microbial Profile Is Altered in Primary Biliary Cholangitis and Partially Restored after Udca Therapy. Gut , 2018. 67 (3) 534-541.

[9] Feld JJ, et al., Abnormal Intestinal Permeability in Primary Biliary Cirrhosis. Dig. Dis. Sci. , 2006. 51 (9) 1607-1613.

[10] Welcker K, et al., Increased Intestinal Permeability in Patients with Inflammatory Bowel Disease.European journal of medical research , 2004. 9 (10) 456-460.

[11] Strowig T, et al., Inflammasomes in Health and Disease. Nature, 2012. 481 (7381) 278-286.

[12] Tsochatzis EA, et al., Liver Cirrhosis. Lancet (London, England), 2014. 383 (9930) 1749-1761.

[13] Murray PJ, et al., Protective and Pathogenic Functions of Macrophage Subsets. Nature reviews. Immunology, 2011. 11 (11) 723-737.

[14] Isaacs-Ten A, et al., Intestinal Microbiome-Macrophage Crosstalk Contributes to Cholestatic Liver Disease by Promoting Intestinal Permeability. Hepatology (Baltimore, Md.) , 2020.

[15] Ma WT, et al., Immunological Abnormalities in Patients with Primary Biliary Cholangitis.Clinical science (London, England : 1979), 2019. 133 (6) 741-760.

[16] You Z, et al., The Immunopathology of Liver Granulomas in Primary Biliary Cirrhosis.J. Autoimmun. , 2012. 39 (3) 216-221.

[17] Leicester KL, et al., Differential Findings for Cd14-Positive Hepatic Monocytes/Macrophages in Primary Biliary Cirrhosis, Chronic Hepatitis C and Nonalcoholic Steatohepatitis. Liver international : official journal of the International Association for the Study of the Liver , 2006. 26 (5) 559-565.

[18] Sasatomi K, et al., Abnormal Accumulation of Endotoxin in Biliary Epithelial Cells in Primary Biliary Cirrhosis and Primary Sclerosing Cholangitis. Journal of hepatology, 1998. 29 (3) 409-416.

[19] Honda Y, et al., Altered Expression of Tlr Homolog Rp105 on Monocytes Hypersensitive to Lps in Patients with Primary Biliary Cirrhosis. Journal of hepatology , 2007. 47 (3) 404-411.

[20] El Kasmi KC, et al., Macrophage-Derived Il-1ß/Nf-Kb Signaling Mediates Parenteral NutritionAssociated Cholestasis. Nature communications , 2018. 9 (1) 1393.

[21] Ma C, et al., Formononetin Attenuates Atherosclerosis Via Regulating Interaction between Klf4 and Sra in Apoe Mice. Theranostics , 2020. 10 (3) 1090-1106.

[22] Yang S, et al., Tectorigenin Attenuates Diabetic Nephropathy by Improving Vascular Endothelium Dysfunction through Activating Adipor1/2 Pathway. Pharmacol. Res. , 2020. 153104678. 
[23] Pan CH, et al., Tectorigenin Inhibits Ifn-Gamma/Lps-Induced Inflammatory Responses in Murine Macrophage Raw 264.7 Cells. Archives of pharmacal research , 2008. 31 (11) 1447-1456.

[24] Gao XX, et al., The Therapeutic Effects of Tectorigenin on Chemically Induced Liver Fibrosis in Rats and an Associated Metabonomic Investigation. Archives of pharmacal research , 2012. 35 (8) 1479-1493.

[25] Lee HU, et al., Hepatoprotective Effect of Tectoridin and Tectorigenin on Tert-Butyl Hyperoxide-Induced Liver Injury. Journal of pharmacological sciences, 2005. 97 (4) 541-544.

[26] Lee HW, et al., Beta-Glucuronidase Inhibitor Tectorigenin Isolated from the Flower of Pueraria Thunbergiana Protects Carbon Tetrachloride-Induced Liver Injury. Liver international : official journal of the International Association for the Study of the Liver, 2003. 23 (4) 221-226.

[27] Fickert P, et al., Characterization of Animal Models for Primary Sclerosing Cholangitis (Psc). Journal of hepatology , 2014. 60 (6) 1290-1303.

[28] Fickert P, et al., A New Xenobiotic-Induced Mouse Model of Sclerosing Cholangitis and Biliary Fibrosis. The American journal of pathology, 2007. 171 (2) 525-536.

[29] Zhang S, et al., Rosiglitazone Alleviates Intrahepatic Cholestasis Induced by A-Naphthylisothiocyanate in Mice: The Role of Circulating 15-Deoxy- $\Delta$-Pgj and Nogo. Br. J. Pharmacol. , 2020. 177 (5) 1041-1060.

[30] Yang S, et al., Formononetin Ameliorates Cholestasis by Regulating Hepatic Sirt1 and Ppara.Biochem. Biophys. Res. Commun. , 2019. 512 (4) 770-778.

[31] Li QY, et al., Tectorigenin Regulates Adipogenic Differentiation and Adipocytokines Secretion Via Ppar and Ikk/Nf-Kb Signaling. Pharm. Biol., 2015. 53 (11) 1567-1575.

[32] Bouhlel MA, et al., Ppargamma Activation Primes Human Monocytes into Alternative M2 Macrophages with Anti-Inflammatory Properties. Cell metabolism , 2007. 6 (2) 137-143.

[33] Hou Y, et al., Pparץ Is an E3 Ligase That Induces the Degradation of Nfxb/P65. Nature communications , 2012. 31300 .

[34] Trauner M, et al., The Rat Canalicular Conjugate Export Pump (Mrp2) Is Down-Regulated in Intrahepatic and Obstructive Cholestasis. Gastroenterology , 1997. 113 (1) 255-264.

[35] Bolder U, et al., Hepatocyte Transport of Bile Acids and Organic Anions in Endotoxemic Rats: Impaired Uptake and Secretion. Gastroenterology, 1997. 112 (1) 214-225.

[36] El Kasmi KC, et al., Phytosterols Promote Liver Injury and Kupffer Cell Activation in Parenteral Nutrition-Associated Liver Disease. Science translational medicine , 2013. 5 (206) 206ra137.

[37] Kaimal R, et al., Differential Modulation of Farnesoid X Receptor Signaling Pathway by the Thiazolidinediones. The Journal of pharmacology and experimental therapeutics , 2009. 330 (1) 125-134.

[38] Halilbasic E, et al., Nuclear Receptors as Drug Targets in Cholestatic Liver Diseases.Clinics in liver disease, 2013. 17 (2) 161-189.

[39] Harada K, et al., Biliary Innate Immunity: Function and Modulation. Mediators of inflammation , 2010. 2010.

[40] Funk C, et al., Cholestatic Potential of Troglitazone as a Possible Factor Contributing to TroglitazoneInduced Hepatotoxicity: In Vivo and in Vitro Interaction at the Canalicular Bile Salt Export Pump (Bsep) in the Rat. Mol. Pharmacol. , 2001. 59 (3) 627-635.

[41] Franca A, et al., Effects of Endotoxin on Type 3 Inositol 1,4,5-Trisphosphate Receptor in Human Cholangiocytes. Hepatology (Baltimore, Md.) , 2019. 69 (2) 817-830.

[42] Li Z, et al., Kmethane-Rich Saline Counteracts Cholestasis-Induced Liver Damage Via Regulating the Tlr4/Nf-B/Nlrp3 Inflammasome Pathway. Oxidative medicine and cellular longevity, 2019. 20196565283. 
[43] Fiorotto R, et al., Loss of Cftr Affects Biliary Epithelium Innate Immunity and Causes Tlr4-Nf-KbMediated Inflammatory Response in Mice.Gastroenterology, 2011. 141 (4) 1498-1508, 1508.e1491-1495.

[44] Miyoshi H, et al., Nf-Kappab Is Activated in Cholestasis and Functions to Reduce Liver Injury. The American journal of pathology, 2001. 158 (3) 967-975.

[45] Luedde T, et al., Ikk1 and Ikk2 Cooperate to Maintain Bile Duct Integrity in the Liver.Proceedings of the National Academy of Sciences of the United States of America, 2008. 105 (28) 9733-9738.

[46] Luedde $\mathrm{T}$, et al., Nf-Kb in the Liver-Linking Injury, Fibrosis and Hepatocellular Carcinoma.Nature reviews. Gastroenterology \& hepatology , 2011. 8 (2) 108-118.

[47] Ahmadian M, et al., PparY Signaling and Metabolism: The Good, the Bad and the Future. Nat. Med. , 2013. 19 (5) 557-566.

[48] Silva AKS, et al., Role of Peroxisome Proliferator-Activated Receptors in Non-Alcoholic Fatty Liver Disease Inflammation. Cellular and molecular life sciences : CMLS , 2018. 75 (16) 2951-2961.

[49] Strautnieks SS, et al., A Gene Encoding a Liver-Specific Abc Transporter Is Mutated in Progressive Familial Intrahepatic Cholestasis. Nat. Genet. , 1998. 20 (3) 233-238.

[50] Xiong XL, et al., Emodin Rescues Intrahepatic Cholestasis Via Stimulating Fxr/Bsep Pathway in Promoting the Canalicular Export of Accumulated Bile. Frontiers in pharmacology, 2019. 10522.

[51] Marrone J, et al., Hepatic Gene Transfer of Human Aquaporin-1 Improves Bile Salt Secretory Failure in Rats with Estrogen-Induced Cholestasis. Hepatology (Baltimore, Md.) , 2016. 64 (2) 535-548.

[52] Funk C, et al., Troglitazone-Induced Intrahepatic Cholestasis by an Interference with the Hepatobiliary Export of Bile Acids in Male and Female Rats. Correlation with the Gender Difference in Troglitazone Sulfate Formation and the Inhibition of the Canalicular Bile Salt Export Pump (Bsep) by Troglitazone and Troglitazone Sulfate. Toxicology, 2001. 167 (1) 83-98.

[53] Han YH, et al., Ror $\alpha$ Induces Klf4-Mediated M2 Polarization in the Liver Macrophages That Protect against Nonalcoholic Steatohepatitis. Cell reports , 2017. 20 (1) 124-135.

[54] Gaul S, et al., Hepatocyte Pyroptosis and Release of Inflammasome Particles Induce Stellate Cell Activation and Liver Fibrosis. Journal of hepatology , 2020. 
Xiang et al, Figure 1

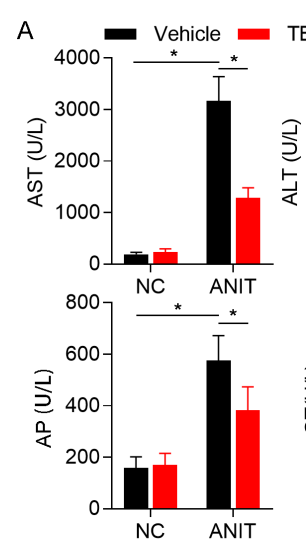

C

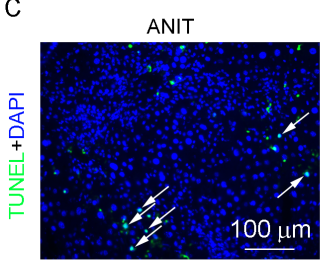

E

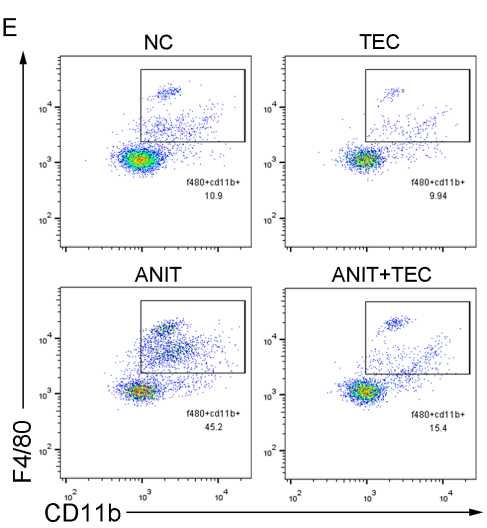

B

NC
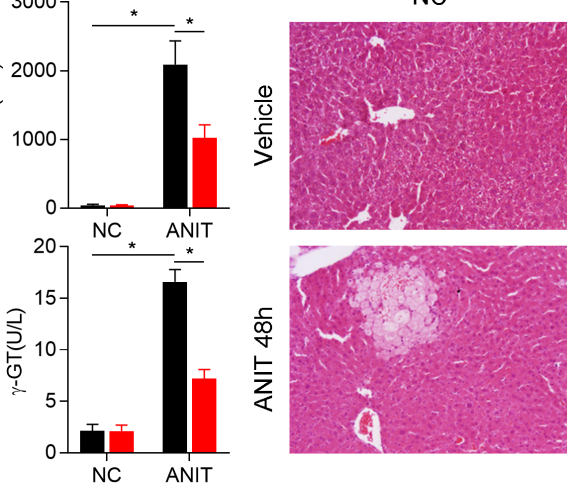

ANIT+TEC
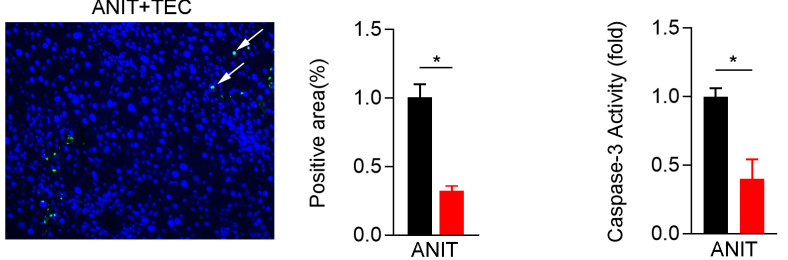

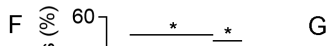

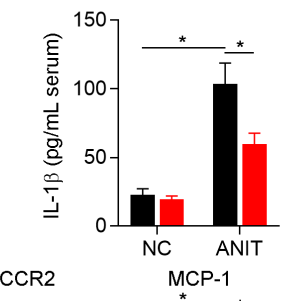

$\mathrm{H} \quad 20 \begin{array}{ll}\text { NC } \\ \text { TNF }-\alpha\end{array}$
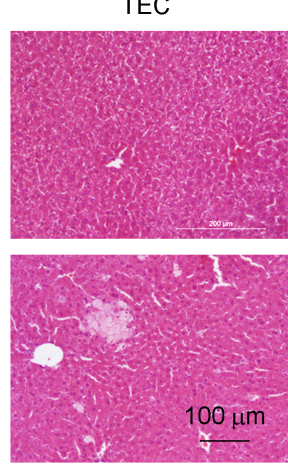

D

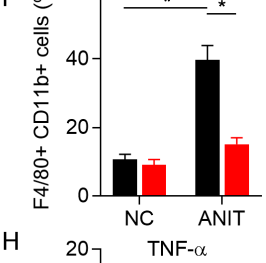

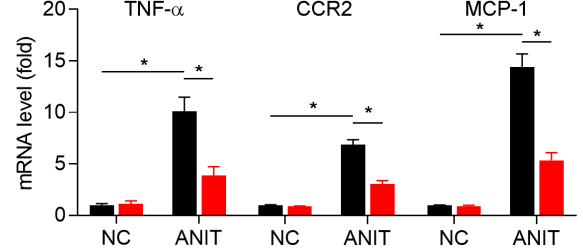


Xiang et al, Figure 2
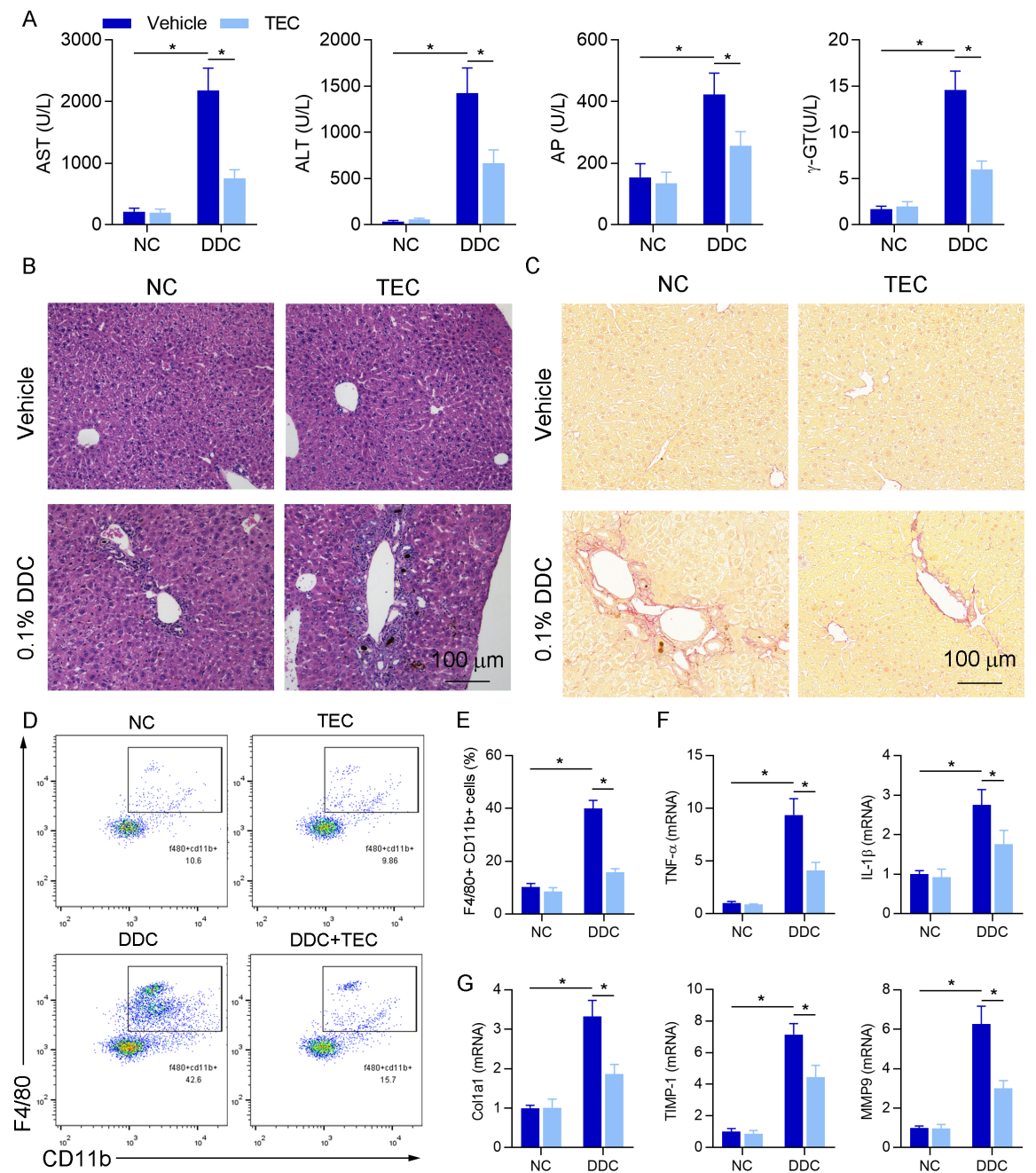

E

F
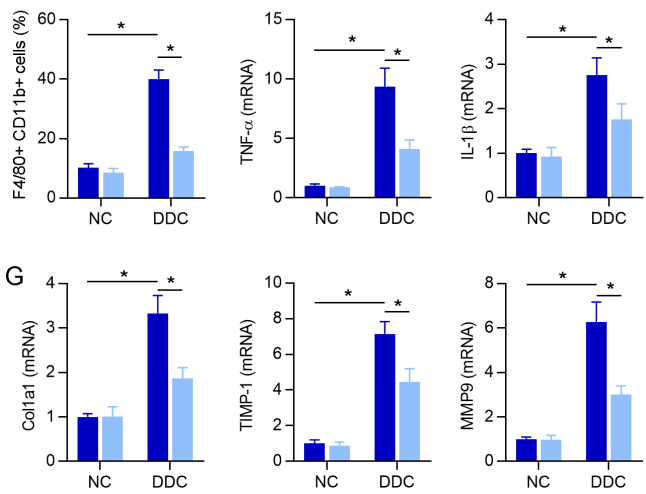
Xiang et al, Figure 3
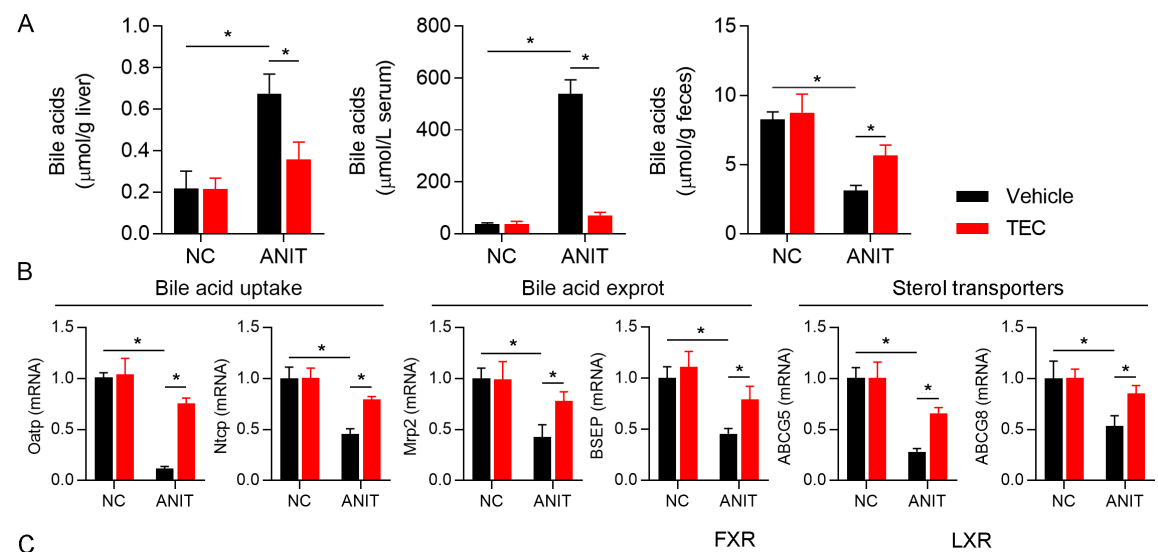

C
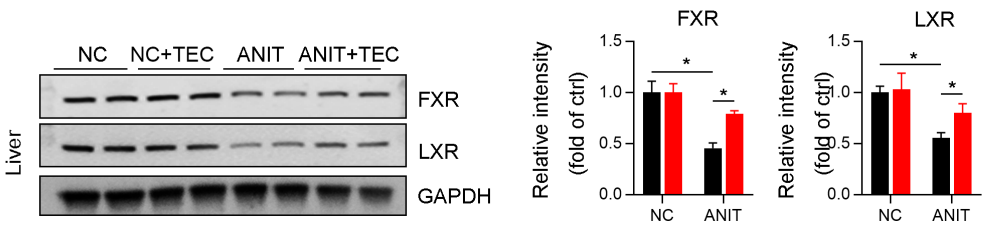

D
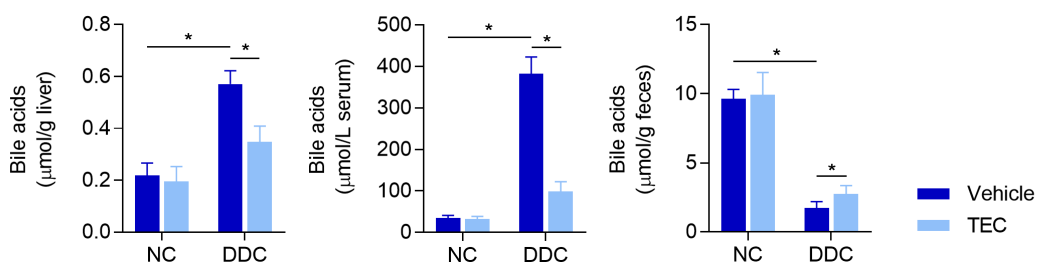

E
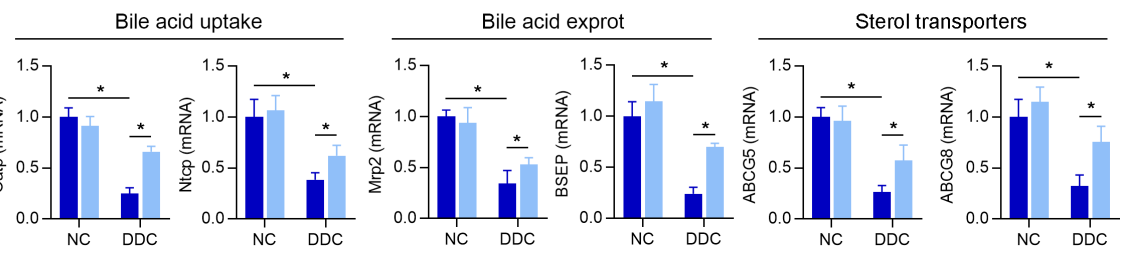

F
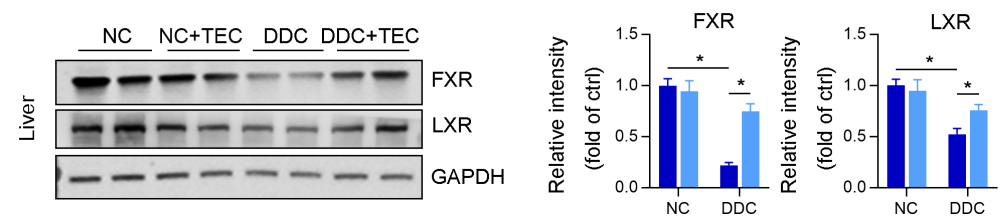
Xiang et al, Figure 4

A

Kupffer Cells
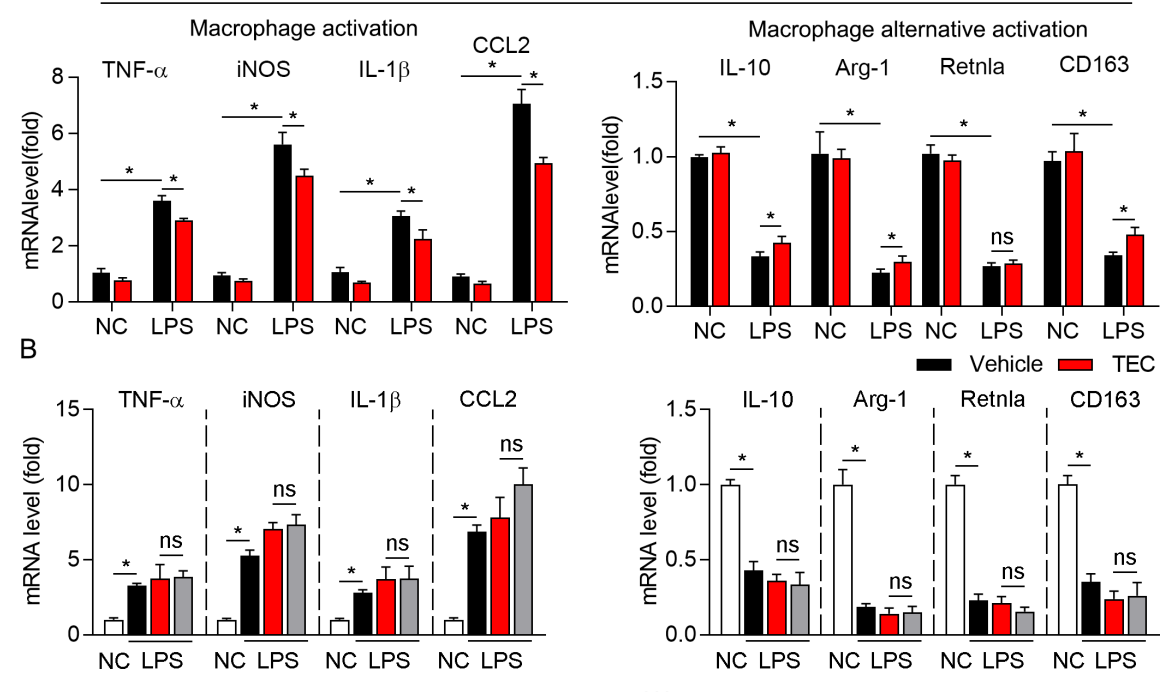

C

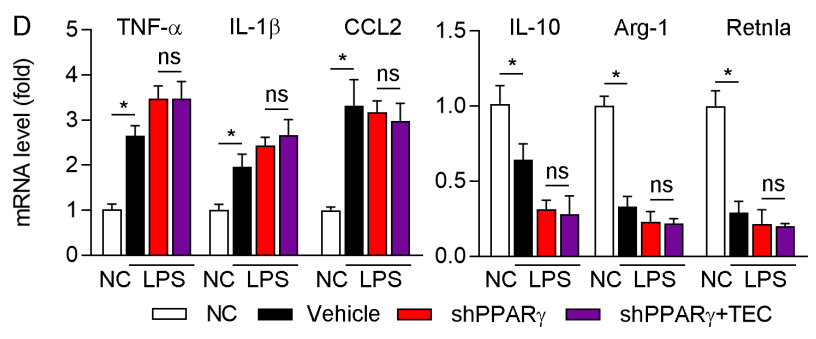


Xiang et al, Figure 5
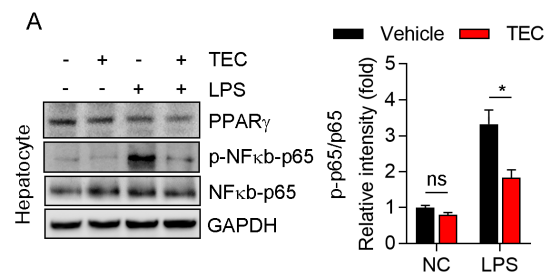

B
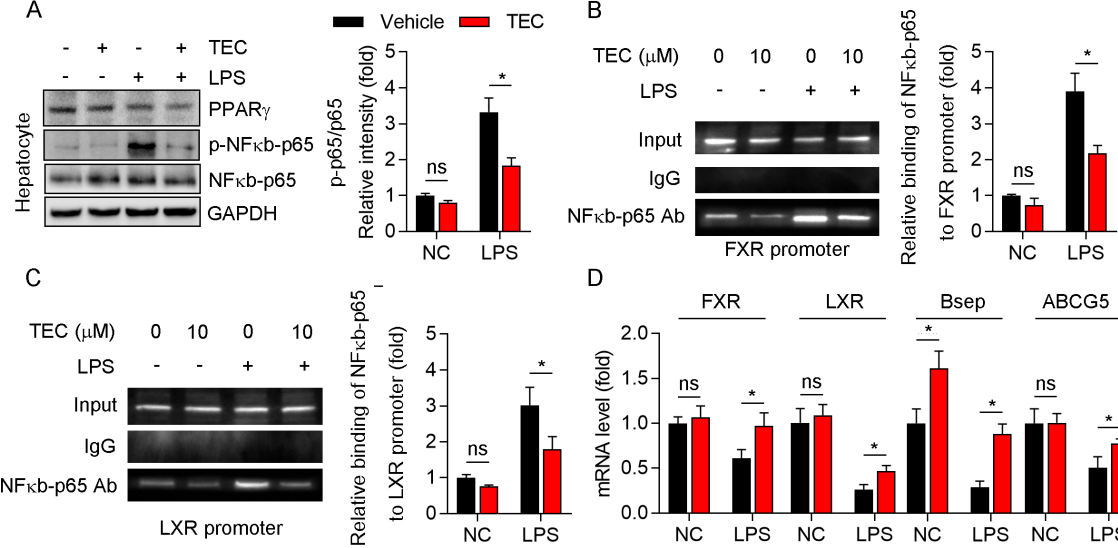

D

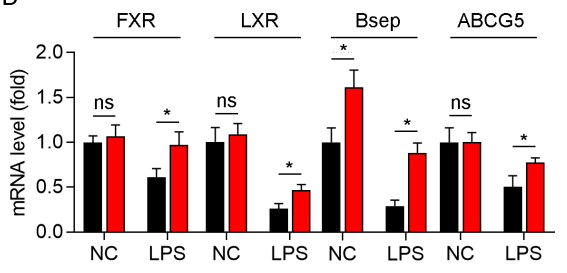

E

$\mathrm{F}$
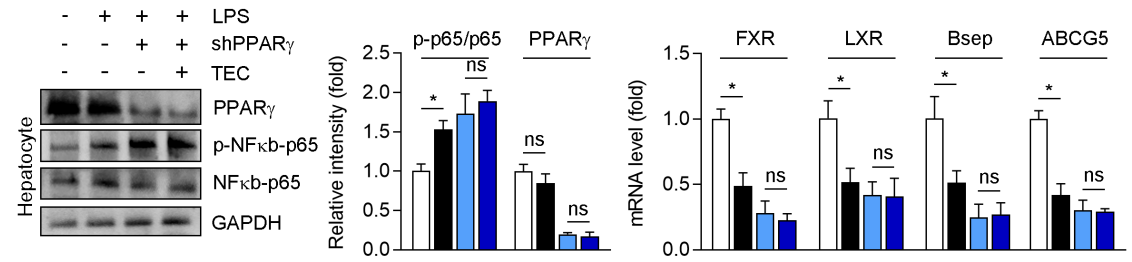

$\square$ NC $\square$ LPS $\square$ shPPAR $\gamma=\operatorname{shPPAR} \gamma+$ TEC

Xiang et al, Figure 6
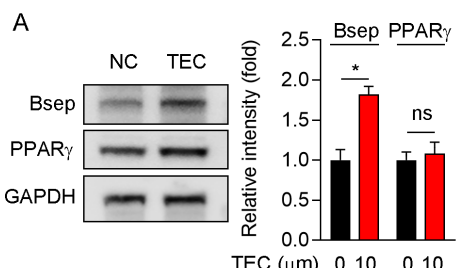

$\operatorname{TEC}(\mu \mathrm{m}) \quad 0 \quad 10 \quad 010$

D

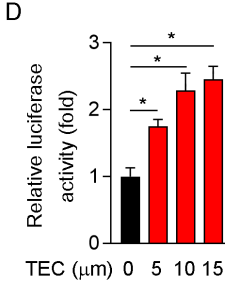

G

E

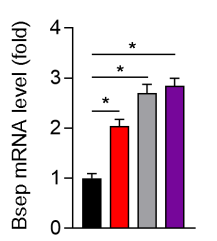

TEC $(\mu \mathrm{m}) 0101010$ TEC $(\mu \mathrm{m}) \quad 0101010$

GW1929 - - + + GW9662 - + +

$\mathrm{F}$
C

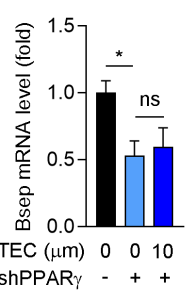

ShPPAR $\gamma++$

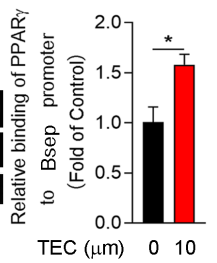

-2753 catagtgtcttgtaaaaagaagatactcaatcagttttgaatgattcaa -2703 agagtaaaataatggcaacttcttttatctcttccatagtgcaatcttca -2653 atatttttaaacaactattggetgaattctaccattgtggtttagagct -2603 ttctctgagacattttgctaaggtttgaaacaggttccaaattgg tcc -2553 acagtgacctccaaggctctacaggagtggtcctcaagcttcagctcatt -2503 gggccccactcccagagtttctgattcagtaggtctcaggaaaggaccaa -2453 gaacatacattcctactgggtaatgaggaggetgctgetggtccagtagt -2403 cacactttcagagccactgccetgtgagctcatggcactccatgattccc

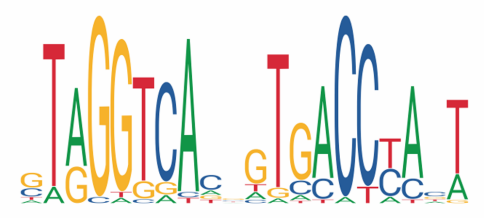


Xiang et al, Figure 7

A B

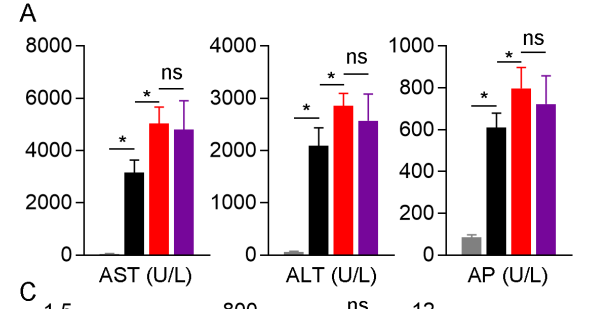

C
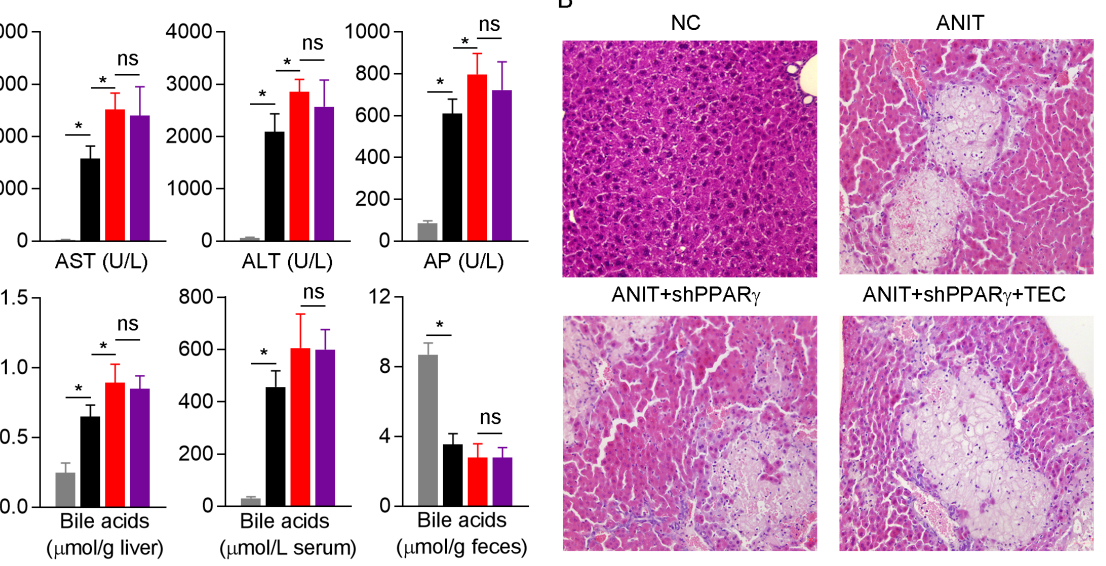

ANIT+shPPAR $\gamma$

ANIT+shPPAR $\gamma+$ TEC
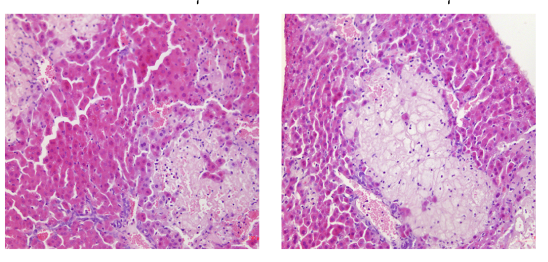

$\square$ NC $\square$ ANIT $=$ ANIT+ShPPAR $\gamma$ ANIT+ShPPAR $\gamma+$ TEC

$\mathrm{D}$

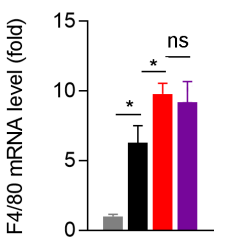

E

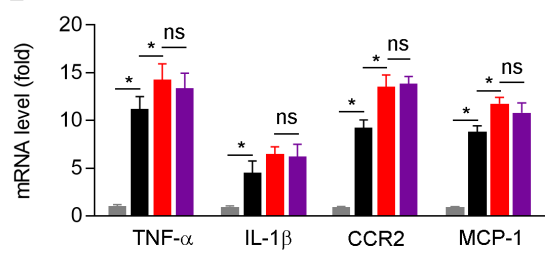

F

G

Bile acid uptake
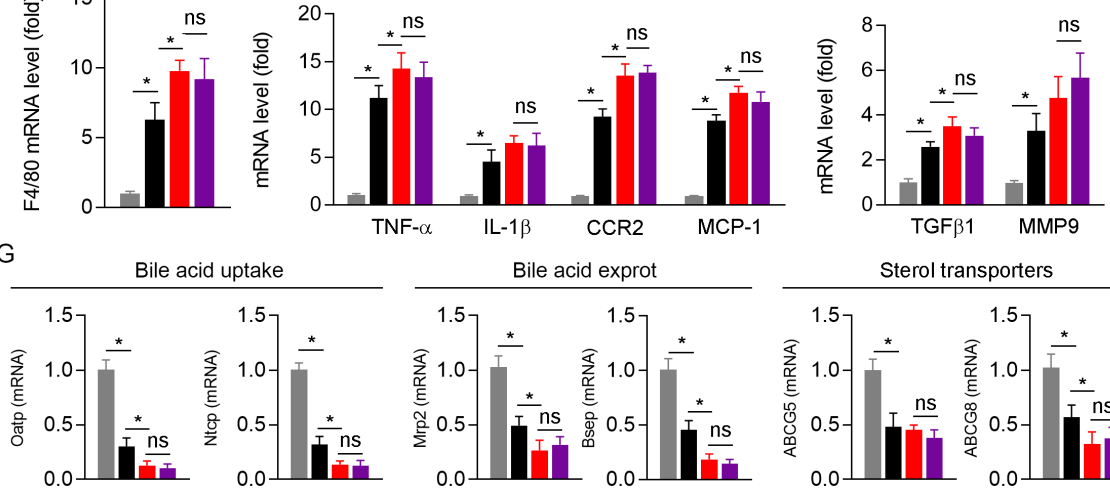

$\mathrm{H}$
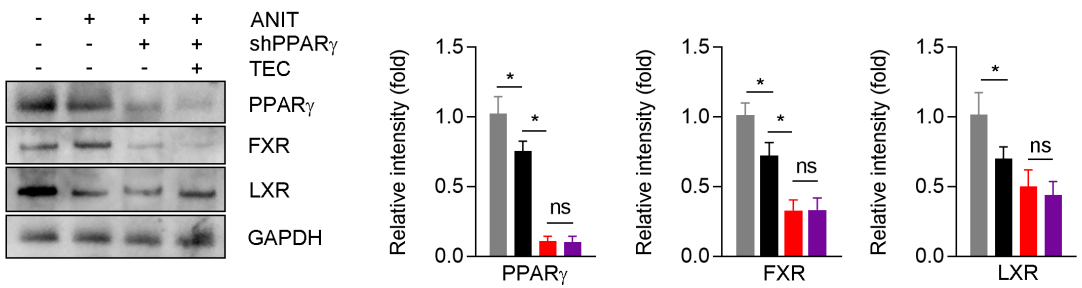
A

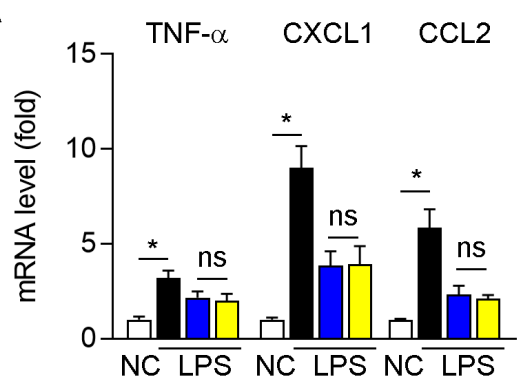

B

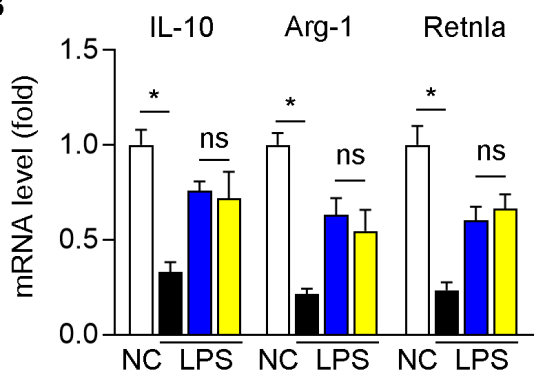

C

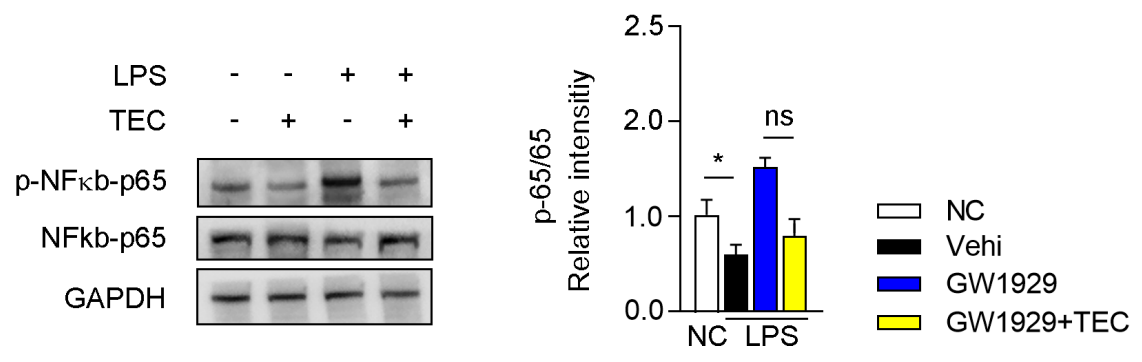

Xiang et al, Figure S2

A

BMDMs
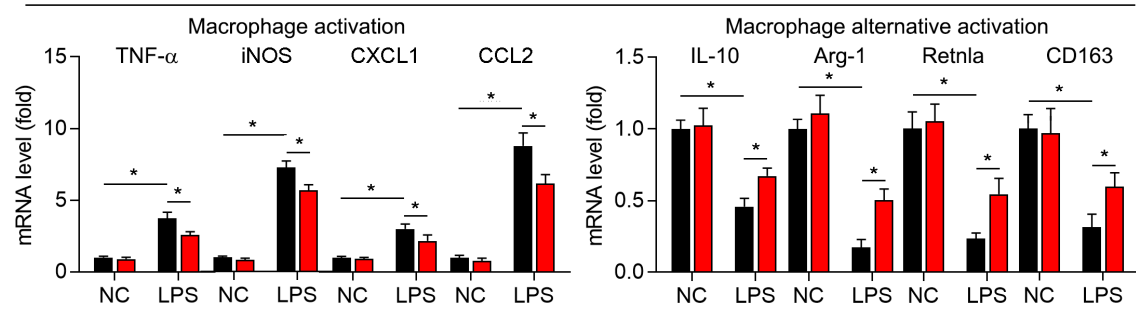

B

$$
\text { - Vehicle } \square \text { TEC }
$$
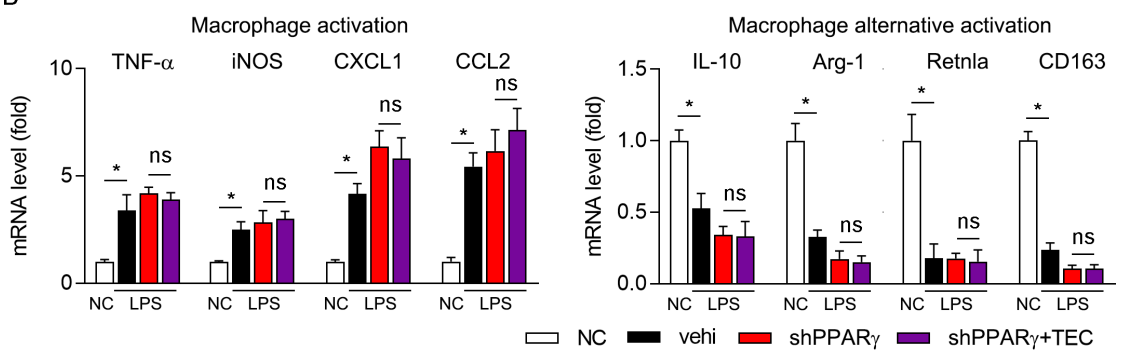

C
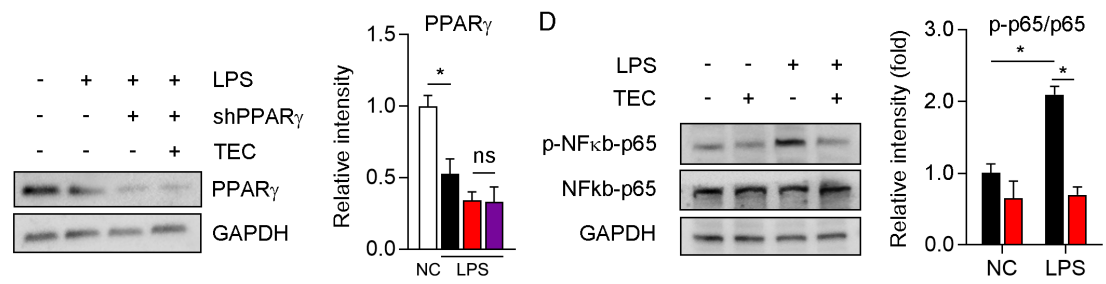
Xiang et al, Figure S3

A
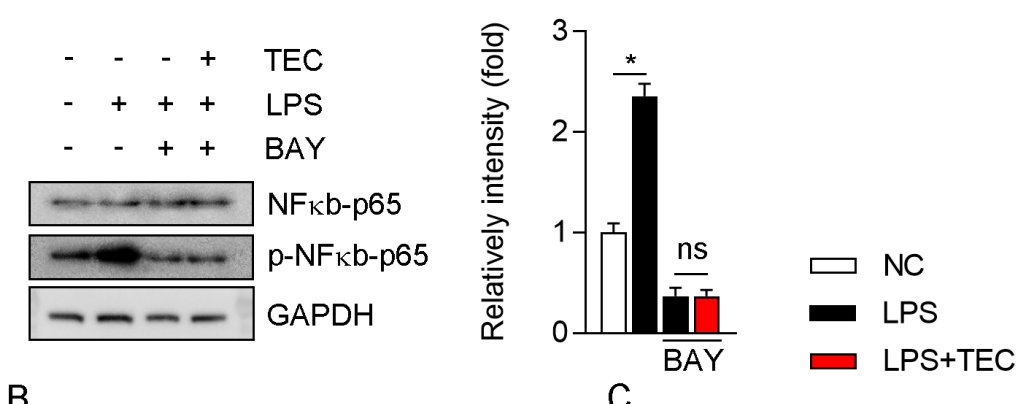

B LXR ABCG5 ABCG8
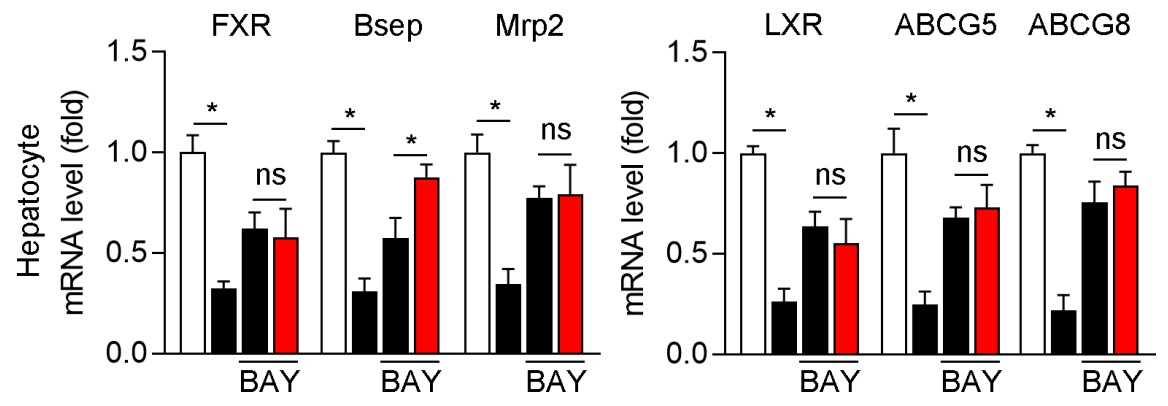


\section{Xiang et al, Figure S4}

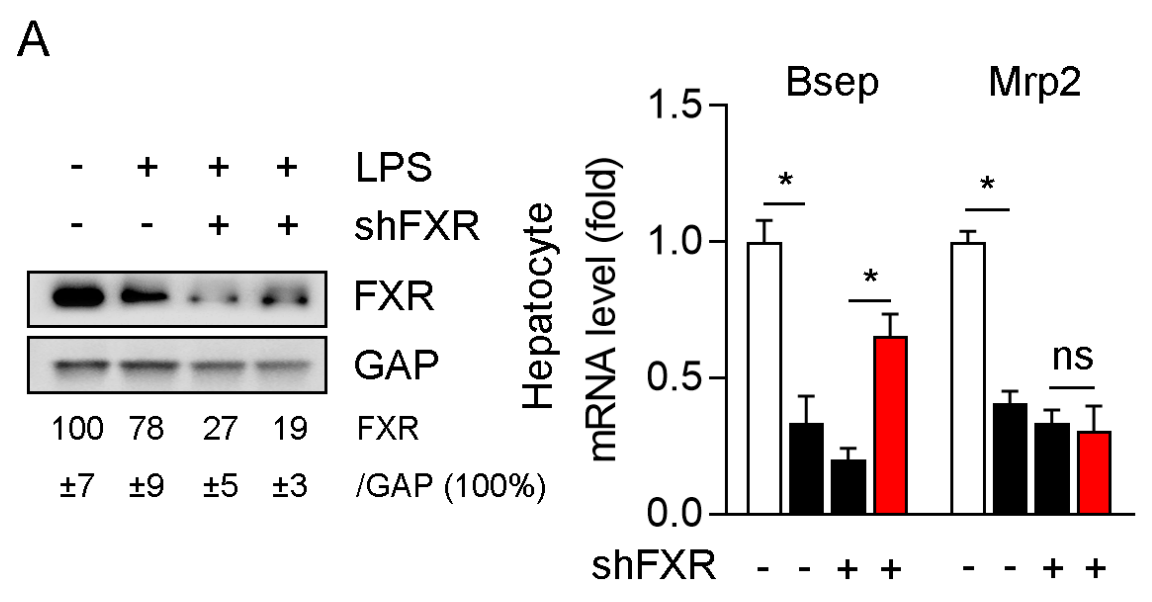

B

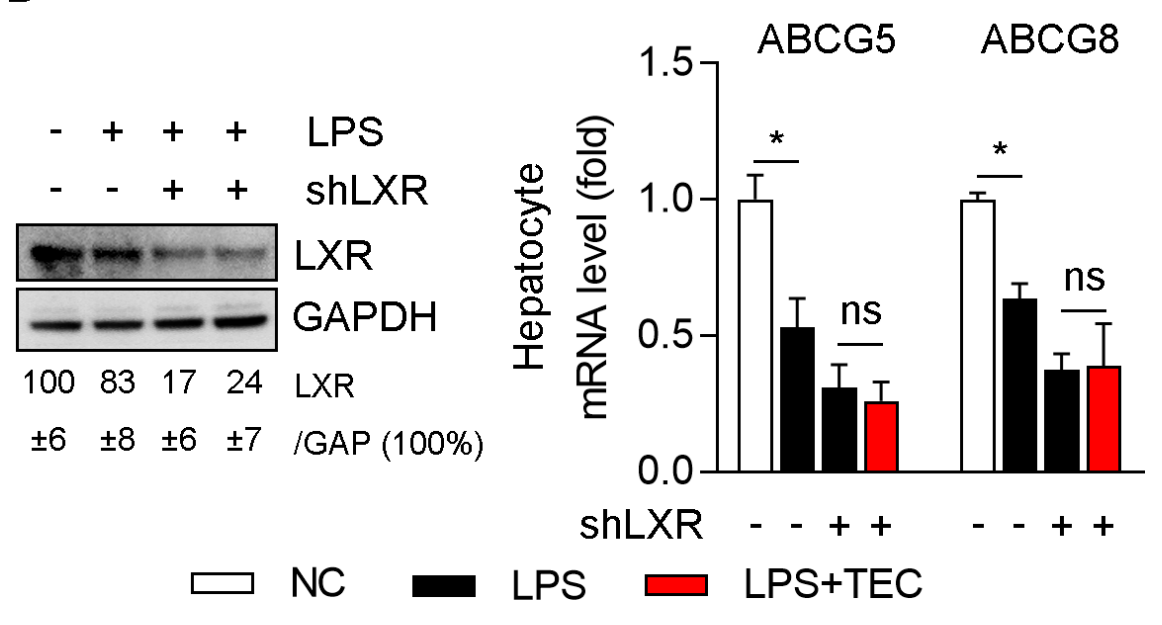

